\title{
EXCHANGEABILITY-TYPE PROPERTIES OF ASSET PRICES
}

\author{
ILYA MOLCHANOV *** AND \\ MICHAEL SCHMUTZ, ${ }^{* * *}$ University of Bern
}

\begin{abstract}
Let $\eta=\left(\eta_{1}, \ldots, \eta_{n}\right)$ be a positive random vector. If its coordinates $\eta_{i}$ and $\eta_{j}$ are exchangeable, i.e. the distribution of $\eta$ is invariant with respect to the swap $\pi_{i j}$ of its $i$ th and $j$ th coordinates, then $\mathrm{E} f(\eta)=\mathrm{E} f\left(\pi_{i j} \eta\right)$ for all integrable functions $f$. In this paper we study integrable random vectors that satisfy this identity for a particular family of functions $f$, namely those which can be written as the positive part of the scalar product $\langle u, \eta\rangle$ with varying weights $u$. In finance such functions represent payoffs from exchange options with $\eta$ being the random part of price changes, while from the geometric point of view they determine the support function of the so-called zonoid of $\eta$. If the expected values of such payoffs are $\pi_{i j}$-invariant, we say that $\eta$ is $i j$-swapinvariant. A full characterisation of the swap-invariance property and its relationship to the symmetries of expected payoffs of basket options are obtained. The first of these results relies on a characterisation theorem for integrable positive random vectors with equal zonoids. Particular attention is devoted to the case of asset prices driven by Lévy processes. Based on this, concrete semi-static hedging techniques for multi-asset barrier options, such as weighted barrier swap options, weighted barrier quanto-swap options, or certain weighted barrier spread options, are suggested.
\end{abstract}

Keywords: Barrier option; duality principle; exchangeability; homogeneous function; Lévy process; multi-asset option; payoff; put-call symmetry; semi-static hedging; symmetry; swap invariance; zonoid

2010 Mathematics Subject Classification: Primary 60E05; 60G51; 91G20

\section{Introduction}

The classic univariate European put-call symmetry property, also known as Bates' rule from [7], relates certain calls and puts in the same market; see, e.g. [6], [11], [22], and, more recently, [14] and [39]. This symmetry property of an integrable random variable $\eta$ can be expressed using expected payoffs from plain vanilla options as

$$
\mathrm{E}(F \eta-k)_{+}=\mathrm{E}(F-k \eta)_{+}
$$

for every strike $k \geq 0$, with $F$ being the forward price, so that the terminal asset price in the one-period setting is $F \eta$ (in order that the discounted expectations can be interpreted as arbitrage-free prices, they have to be taken with respect to a martingale measure); see [14]

Received 11 May 2010; revision received 4 January 2011.

* Postal address: Department of Mathematical Statistics and Actuarial Science, University of Bern, Sidlerstrasse 5, 3012 Bern, Switzerland.

Supported by the Swiss National Science Foundation, under grants 200021-117606 and 200021-126503.

** Email address: ilya.molchanov@ stat.unibe.ch

*** Email address: michael.schmutz@stat.unibe.ch 
and [30]. In cases with vanishing carrying costs the put-call symmetry makes it possible to replace at certain times a call option with equally valued puts in order to design so-called semistatic hedges for barrier options. In cases of nonvanishing carrying costs semi-static hedges can be constructed on the basis of a very closely related property, called quasi-self-duality, briefly discussed in Section 6; see also [14] and [30].

Following Carr and Lee [14], semi-static hedging is the replication of contracts by trading European-style claims no more than twice after inception. In the single-asset case such semistatic hedging strategies have been analysed extensively in recent years; see, e.g. [2], [3], [9], [12], [16], and, more recently, [14].

Interestingly, the duality principle in option pricing also traces some of its roots to the same papers as put-call symmetry results; see, e.g. [6], [7], [11], and [24]. The power of duality lies in the possibility to reduce the complexity of valuation problems by relating them to easier problems in the dual markets. For a presentation of this principle in a general univariate exponential semimartingale setting, see [17]; for bivariate Lévy markets, see [20]; for multivariate semi-martingale extensions (with various dual markets), see [18]. The symmetry property then appears if the original and certain dual markets coincide, motivating the name self-dual chosen in [30] for distributions that coincide with their duals.

In the multi-asset setting $\eta=\left(\eta_{1}, \ldots, \eta_{n}\right)$ is an $n$-dimensional random vector with positive coordinates such that the price $S_{T i}$ of the $i$ th asset at time $T>0$ equals $F_{i} \eta_{i}$, where, in a risk-neutral world, $F_{i}$ stands for the corresponding theoretical forward price and $\eta_{i}$ denotes the random part of the price change of the $i$ th asset. We define

$$
S_{T}=\left(S_{T 1}, \ldots, S_{T n}\right)=\left(F_{1} \eta_{1}, \ldots, F_{n} \eta_{n}\right)=F \circ \eta .
$$

Furthermore, assume that $\mathrm{Q}$ is a probability measure that makes $\eta$ integrable. For later applications to barrier options, we also assume that $\mathrm{Q}$ is a martingale measure that is consistent with market option prices. The expectation with respect to $\mathrm{Q}$ is denoted by E. For further ease of notation, we omit the time subscript $T$ on $\eta$ and, for the moment, incorporate the forward prices $F_{i}, i=1, \ldots, n$, into the payoff functions. In our context payoff functions are measurable functions $f:(0, \infty)^{n} \mapsto \mathbb{R}_{+}$.

Molchanov and Schmutz [30] studied symmetries of expected payoffs from European basket options defined as

$$
f_{\mathrm{b}}\left(u_{0}, u_{1}, \ldots, u_{n}\right)=\left(\sum_{l=1}^{n} u_{l} \eta_{l}+u_{0}\right)_{+}, \quad u_{0}, u_{1}, \ldots, u_{n} \in \mathbb{R} .
$$

When writing the 'weights' of a basket option together with its strike as a vector, we number the coordinates of the obtained $(n+1)$-dimensional vectors as $0,1, \ldots, n$ and denote these vectors as $\left(u_{0}, u\right)$ for $u_{0} \in \mathbb{R}$ and $u \in \mathbb{R}^{n}$ or as $\left(u_{0}, u_{1}, \ldots, u_{n}\right)=\left(u_{0}, u\right) \in \mathbb{R}^{n+1}$. In the following we consider vectors as rows or columns, depending on the situation.

Since $f_{\mathrm{b}}\left(u_{0}, u\right)$ can be understood as a plain vanilla option on the scalar product $\langle u, \eta\rangle$ with strike $u_{0}$, the corresponding expected payoffs uniquely determine the distribution of $\langle u, \eta\rangle$ (see, e.g. [10] and [34]), and thereupon also determine the distribution of $\eta$ as the following result (which holds also for not necessarily positive $\eta$ ) shows. Note that the expected values of $f_{\mathrm{b}}\left(u_{0}, u\right)$ considered as a function of $\left(u_{0}, u\right)$ constitute the support function of an $(n+1)$ dimensional convex body called the lift zonoid of $\eta$; see [31, Section 2.2].

Theorem 1.1. (See, e.g. [30] and [31].) The expected values $\mathrm{E} f_{\mathrm{b}}\left(u_{0}, u_{1}, \ldots, u_{n}\right)$ for all $u_{0} \in \mathbb{R}$ and $u \in \mathbb{R}^{n}$ uniquely determine the distribution of an integrable random vector $\eta$. 
Although it is possible to weaken the statement of Theorem 1.1 by considering only one fixed $u_{0} \neq 0$, the uniqueness does not hold any more if $u_{0}=0$, i.e. for the payoffs from swap (or exchange) options defined as

$$
f_{\mathrm{b}}^{\mathrm{o}}(u)=\left(\sum_{l=1}^{n} u_{l} \eta_{l}\right)_{+}=(\langle u, \eta\rangle)_{+}, \quad u \in \mathbb{R}^{n} .
$$

The random vector $\eta$ with positive coordinates is called self-dual with respect to the $i$ th numeraire if $\eta$ is integrable and $\mathrm{E} f_{\mathrm{b}}\left(u_{0}, u_{1}, \ldots, u_{n}\right)$ as a function of $\left(u_{0}, u\right)$ is invariant with respect to the permutation of $u_{0}$ and the $i$ th coordinate of $u$; see [30, Section 2]. A jointly self-dual $\eta$ satisfies this property for all numeraires $i=1, \ldots, n$, so that the expected payoff $\mathrm{E} f_{\mathrm{b}}\left(u_{0}, u_{1}, \ldots, u_{n}\right)$ becomes symmetric in all its $n+1$ arguments. This joint self-duality property implies that $\eta$ is exchangeable, i.e. $\left(\eta_{1}, \ldots, \eta_{n}\right)$ coincides in distribution with $\left(\eta_{l_{1}}, \ldots, \eta_{l_{n}}\right)$ for each permutation of its components. The exchangeability property is well studied in probability theory; see, e.g. [1] or [26] and the literature cited therein. It is also known from [30, Section 3] that the exchangeability property is strictly weaker than the joint self-duality.

While the self-duality property is crucial to switch between put and call options as in (1.1), hedges for some other derivatives do not rely on the self-duality assumption. In particular, this relates to derivatives with the payoff function (1.2). For example, we can require that

$$
\mathrm{E}\left(u_{1} \eta_{1}+u_{2} \eta_{2}\right)_{+}=\mathrm{E}\left(u_{1} \eta_{2}+u_{2} \eta_{1}\right)_{+}
$$

for every $\left(u_{1}, u_{2}\right) \in \mathbb{R}^{2}$ in the two-asset case. This swap-invariance property is weaker than the exchangeability of $\eta$, e.g. it will be shown later that in the risk-neutral setting each twodimensional log-normally distributed random vector satisfies (1.3), no matter that its coordinates are not identically distributed and so are not exchangeable unless the two assets share the same volatility. This property helps to design semi-static hedges for certain barrier options, e.g. the knock-out contract with payoff defined by

$$
\left(a S_{T 1}-b S_{T 2}\right)_{+} 1_{\left\{c S_{t 1}>S_{t 2} \text { for all } t \in[0, T]\right\}}
$$

where $1_{\{\cdot\}}$ denotes the indicator function, and $S_{t 1}$ and $S_{t 2}, t \in[0, T]$, are two price processes (with equal carrying costs); for details, see Section 7, in particular Example 7.2.

We proceed with a concise discussion of the $i j$-exchangeability property in Section 2 . In Section 3 we characterise the weaker swap-invariance property and discuss its relationships to self-duality. Weighted variants of the swap invariance are considered in Section 4. In Section 5 we analyse log-infinitely divisible distributions exhibiting the swap-invariance property. The necessity to handle unequal carrying costs in important applications motivates further weakening of the swap-invariance property in Section 6. Finally, in Section 7 we present applications for creating semi-static hedges for certain multi-asset derivatives with knocking conditions. The development of semi-static replication strategies of multi-asset barrier options (see Section 7) and possibly also more complicated path-dependent contracts is probably the most important application of exchangeability-type properties in finance. The importance of developing robust hedging strategies for multi-asset path-dependent financial derivatives is particularly stressed by Carr and Laurence [13]. Other obvious applications of the described symmetry results may be found in the area of validating models or analysing market data, e.g. extending the univariate case considered in [7] and [21]. 


\section{Exchangeable random vectors}

For each $i, j \in\{1, \ldots, n\}, i \neq j$, define a linear mapping on $\mathbb{R}^{n}$ by

$$
\pi_{i j}(x)=\left(x_{1}, \ldots, x_{i-1}, x_{j}, x_{i+1}, \ldots, x_{j-1}, x_{i}, x_{j+1}, \ldots, x_{n}\right),
$$

i.e. $\pi_{i j}$ transposes (swaps) the $i$ th and $j$ th coordinates of $x$. If the distribution of a random vector $\eta$ in $\mathbb{R}^{n}$ is $\pi_{i j}$-invariant, we say that $\eta$ is $i j$-exchangeable. The following result follows directly from Theorem 1.1.

Corollary 2.1. An integrable random vector $\eta$ is $i j$-exchangeable if and only if $\mathrm{E} f_{\mathrm{b}}\left(u_{0}, u\right)$ is invariant with respect to permutation of the ith and $j$ th coordinates of $u$ for all $u \in \mathbb{R}^{n}$ and any fixed $u_{0} \neq 0$.

In view of financial applications, assume that all coordinates of $\eta$ are positive, so that $\eta=\mathrm{e}^{\xi}$ for a random vector $\xi=\left(\xi_{1}, \ldots, \xi_{n}\right)$, where the exponential function is applied coordinatewise. Because of the widespread use of Lévy models for derivative pricing, we characterise infinitely divisible random vectors $\xi=\log \eta$ for $i j$-exchangeable $\eta$. In the sequel we denote the Euclidean norm by $\|\cdot\|$, the imaginary unit $\sqrt{-1}$ by $\mathrm{i}$, and use the following formulation of the LévyKhintchine formula for the characteristic function of $\xi$ (see [35, Chapter 2]):

$$
\begin{aligned}
\varphi_{\xi}(u) & =\operatorname{Ee}^{\mathrm{i}\langle u, \xi\rangle} \\
& =\exp \left\{\mathrm{i}\langle\gamma, u\rangle-\frac{1}{2}\langle u, A u\rangle+\int_{\mathbb{R}^{n}}\left(\mathrm{e}^{\mathrm{i}\langle u, x\rangle}-1-\mathrm{i}\langle u, x\rangle 1_{\{\|x\| \leq 1\}}\right) \mathrm{d} \nu(x)\right\}, \quad u \in \mathbb{R}^{n} .
\end{aligned}
$$

Here $A$ is a symmetric nonnegative definite $n \times n$ matrix, $\gamma \in \mathbb{R}^{n}$ is a constant vector, and $v$ is a Lévy measure on $\mathbb{R}^{n}$, namely $v(\{0\})=0$ and

$$
\int_{\mathbb{R}^{n}} \min \left(\|x\|^{2}, 1\right) \mathrm{d} v(x)<\infty .
$$

Since the $i j$-exchangeability of $\xi$ is equivalent to the $\pi_{i j}$-invariance of its characteristic function, we immediately obtain the following result.

Proposition 2.1. Let $\eta=\mathrm{e}^{\xi}$ with $\xi$ being infinitely divisible. Then $\eta$ is ij-exchangeable if and only if the generating triplet $(A, v, \gamma)$ of $\xi$ satisfies the following conditions.

(a) The matrix $A=\left(a_{l m}\right)_{l m=1}^{n}$ satisfies $a_{i i}=a_{j j}$ and $a_{l i}=a_{l j}$ for all $l=1, \ldots, n, l \neq i, j$.

(b) The Lévy measure is $\pi_{i j}$-invariant, i.e. $v(B)=v\left(\pi_{i j} B\right)$ for all Borel $B$.

(c) The ith and jth coordinates of $\gamma$ coincide.

Example 2.1. (Log-normal distribution, Black-Scholes setting.) Assume that $\eta=\mathrm{e}^{\xi}$ is $\log$ normal with $\xi$ having expectation $\mu$ and covariance matrix $A$. Then $\eta$ is $i j$-exchangeable if and only if $A$ satisfies $a_{i i}=a_{j j}$ and $a_{l i}=a_{l j}$ for $l=1, \ldots, n, l \neq i, j$ (with the remaining $a_{l m}$ arbitrarily chosen such that $A$ is nonnegative definite), and $\mu_{i}=\mu_{j}$. The latter automatically holds if all components of $\eta$ are related to a martingale measure, i.e. $\mu=-\frac{1}{2}\left(a_{11}, \ldots, a_{n n}\right)$. In bivariate risk-neutral cases the only restriction is the equality of the variances, while the correlation coefficient between $\xi_{1}$ and $\xi_{2}$ can be arbitrary. 


\section{Swap invariance}

Now we consider the symmetry property for the payoff function (1.2).

Definition 3.1. An integrable random vector $\eta$ with positive components is said to be $i j$-swapinvariant if the expected value $\mathrm{E} f_{\mathrm{b}}^{\mathrm{O}}(u)$ is invariant with respect to swapping the $i$ th and $j$ th coordinates of any $u \in \mathbb{R}^{n}$.

This property yields $\mathrm{E} \eta_{i}=\mathrm{E} \eta_{j}$, which is clearly weaker than the $i j$-exchangeability of $\eta$.

In the following we often need to change the probability measure Q. Let $\eta=\mathrm{e}^{\xi}$, and let $\zeta$ be a random variable that together with $\xi$ builds the $(n+1)$-dimensional random vector $(\xi, \zeta)$. If $\mathrm{e}^{\langle w,(\xi, \zeta)\rangle}$ with $w \in \mathbb{R}^{n+1}$ is integrable, define $\mathrm{Q}^{w}$ by

$$
\frac{\mathrm{dQ}^{w}}{\mathrm{dQ}}=\frac{\mathrm{e}^{\langle w,(\xi, \zeta)\rangle}}{\mathrm{E}^{\langle w,(\xi, \zeta)\rangle}}
$$

i.e. $\mathbf{Q}^{w}$ is the Esscher transform of $\mathbf{Q}$ with parameter $w$. In the case where $w \in \mathbb{R}^{n}$, the same notation applies with $w$ extended by a zero component. If $w=e_{j}$ is the $j$ th standard basis vector in $\mathbb{R}^{n+1}$ then we write $\mathrm{Q}^{j}$ for short. The expectation with respect to changed measures is indicated by the corresponding subscript.

The following result shows that $i j$-swap-invariance is related to the self-duality in a lowerdimensional space. Define the functions $\tilde{\kappa}_{j}:(0, \infty)^{n} \mapsto(0, \infty)^{n-1}$ acting as

$$
\tilde{\kappa}_{j}(x)=\left(\frac{x_{1}}{x_{j}}, \ldots, \frac{x_{j-1}}{x_{j}}, \frac{x_{j+1}}{x_{j}}, \ldots, \frac{x_{n}}{x_{j}}\right), \quad j=1, \ldots, n .
$$

Theorem 3.1. Let $\eta$ be integrable and $i, j \in\{1, \ldots, n\}, i<j$. Then the following two statements are equivalent.

(a) The n-dimensional random vector $\eta$ is ij-swap-invariant under $\mathrm{Q}$.

(b) The $(n-1)$-dimensional random vector $\tilde{\kappa}_{j}(\eta)$ is self-dual with respect to the ith numeraire under the probability measure $\mathrm{Q}^{j}$.

Proof. The change-of-measure formula yields, for all $u \in \mathbb{R}^{n}$,

$$
\begin{gathered}
\mathrm{E}_{\mathrm{Q}^{j}}\left(\sum_{l=1, l \neq j}^{n} u_{l} \frac{\eta_{l}}{\eta_{j}}+u_{j}\right)_{+}=\left(\mathrm{E} \eta_{j}\right)^{-1} \mathrm{E}\left(\sum_{l=1}^{n} u_{l} \eta_{l}\right)_{+}, \\
\mathrm{E}_{\mathrm{Q}^{j}}\left(\sum_{l=1, l \neq i, j}^{n} u_{l} \frac{\eta_{l}}{\eta_{j}}+u_{i}+u_{j} \frac{\eta_{i}}{\eta_{j}}\right)_{+}=\left(\mathrm{E} \eta_{j}\right)^{-1} \mathrm{E}\left(\sum_{l=1, l \neq i, j}^{n} u_{l} \eta_{l}+u_{i} \eta_{j}+u_{j} \eta_{i}\right)_{+} .
\end{gathered}
$$

The equality of the right-hand sides characterises the $i j$-swap-invariance of $\eta$, while the equality of the left-hand sides means the self-duality of $\tilde{\kappa}_{j}(\eta)$ with respect to the $i$ th numeraire under $\mathrm{Q}^{j}$.

Remark 3.1. In view of Corollary 2.1 we can show by a similar argument that if $n \geq 3$ and $i, j<k$ (for notational convenience), then Theorem 3.1(a) holds if and only if $\tilde{\kappa}_{k}(\eta)$ is $i j$ exchangeable under $\mathrm{Q}^{k}$. In the risk-neutral foreign exchange setting $\mathrm{Q}^{k}$ acquires an immediate interpretation in the market where trades take place in the currency number $k$. 
Example 3.1. (Bivariate swap invariance and symmetry.) Let $\eta$ be a bivariate swap-invariant random vector with $\mathrm{E} \eta_{1}=1$. Then $\tilde{\kappa}_{1}(\eta)=\eta_{2} / \eta_{1}$ is denoted by $\tilde{\eta}$ and

$$
\mathrm{E}_{\mathrm{Q}^{1}}\left(u_{1} \tilde{\eta}+u_{2}\right)_{+}=\mathrm{E}\left(u_{1} \eta_{2}+u_{2} \eta_{1}\right)_{+}=\mathrm{E}\left(u_{1} \eta_{1}+u_{2} \eta_{2}\right)_{+}=\mathrm{E}_{\mathrm{Q}^{1}}\left(u_{1}+u_{2} \tilde{\eta}\right)_{+}
$$

for all $u_{1}, u_{2} \in \mathbb{R}$. Hence, $\left(\eta_{1}, \eta_{2}\right)$ is swap invariant under $Q$ if and only if $\tilde{\eta}$ satisfies the classical univariate European put-call symmetry under the 'dual-market' measure $\mathrm{Q}^{1}$. In particular, this means that bivariate swap invariance is not more restrictive than the very well-known and often applied European put-call symmetry. For the analysis and characterisation of even weaker properties, we refer the reader to Section 6.

The expected payoff $\mathrm{E} f_{\mathrm{b}}^{\mathrm{o}}(u)$ as a function of $u$ becomes the support function of an $n$-dimensional convex body called the zonoid of $\eta$; see, e.g. [31, Section 2.1] for a detailed discussion about these well-known convex bodies in relation to random vectors. In particular, it is well known that zonoids (unlike lift zonoids from Theorem 1.1) do not uniquely characterise the distribution of $\eta$.

The $i j$-swap-invariance of $\eta$ means that its zonoid is symmetric with respect to the plane $\left\{u_{i}=u_{j}\right\}$, or, equivalently, that $\eta$ and $\pi_{i j}(\eta)$ share the same zonoid. In view of this, we first characterise general positive integrable random vectors with equal zonoids. In the following we let $\mathbf{1}=(1, \ldots, 1)$ in the space of an appropriate dimension.

Theorem 3.2. Let $\eta=\mathrm{e}^{\xi}$ and $\eta^{*}=\mathrm{e}^{\xi^{*}}$ be integrable random vectors. Then

$$
\mathrm{E}(\langle u, \eta\rangle)_{+}=\mathrm{E}\left(\left\langle u, \eta^{*}\right\rangle\right)_{+} \text {for all } u \in \mathbb{R}^{n}
$$

if and only if

$$
\varphi_{\xi}(u-\mathrm{i} w)=\varphi_{\xi}(u-\mathrm{i} w)
$$

for all $u \in \mathbb{H}$, where

$$
\mathbb{H}=\left\{u \in \mathbb{R}^{n}: \sum_{k=1}^{n} u_{k}=0\right\},
$$

and at least one (and then necessarily for all) $w$ such that $\sum w_{i}=1$ and both sides of (3.3) are finite.

Proof. Necessity. Equality (3.2) implies that $\mathrm{E} \eta_{i}=\mathrm{E} \eta_{i}^{*}$ for all $i$. Change the measure $\mathrm{Q}$ to $\mathrm{Q}^{1}$ and $\mathrm{Q}^{1 *}$ using $\eta_{1}$ and $\eta_{1}^{*}$, respectively, as the density normalised by the expectation. By Theorem 1.1, the distribution of $\tilde{\kappa}_{1}(\eta)$ under $\mathrm{Q}^{1}$ coincides with the distribution of $\tilde{\kappa}_{1}\left(\eta^{*}\right)$ under $\mathrm{Q}^{1 *}$. Assume that (3.3) is finite, i.e. $\mathrm{Ee}^{\langle w, \xi\rangle}<\infty$ for some $w \in \mathbb{R}^{n}$ with $\sum w_{i}=1$. Then

$$
f(\xi)=\exp \left\{\mathrm{i}\left\langle\left(u_{2}, \ldots, u_{n}\right)-\mathrm{i}\left(w_{2}, \ldots, w_{n}\right),\left(\xi_{2}-\xi_{1}, \ldots, \xi_{n}-\xi_{1}\right)\right\rangle\right\}
$$

is integrable under $\mathrm{Q}^{1}$, so that $f\left(\xi^{*}\right)$ is integrable under $\mathrm{Q}^{1 *}$ and both expectations are equal. By changing the measures back and using $u=\left(-\sum_{i=2}^{n} u_{i}, u_{2}, \ldots, u_{n}\right) \in \mathbb{H}$ and $\sum_{k=1}^{n} w_{k}=1$, this implies (3.3).

Sufficiency. If the both sides of (3.3) are finite and equal for some $w$, then

$$
\mathrm{Ee}^{\langle w, \xi\rangle}=\mathrm{Ee}^{\left\langle w, \xi^{*}\right\rangle}=c .
$$

Thus, the characteristic functions (restricted on $\mathbb{H}$ ) of $\xi$ under the changed measure $\mathrm{Q}^{w}$ and of $\xi^{*}$ under $\mathrm{Q}^{w *}$ coincide, where the change of measure is done with the normalised densities $\mathrm{e}^{\langle w, \xi\rangle}$ 
and $\mathrm{e}^{\left\langle w, \xi^{*}\right\rangle}$, respectively. Therefore, $\xi-\mathbf{1} \xi_{1}$ under $\mathrm{Q}^{w}$ is identically distributed as $\xi^{*}-\mathbf{1} \xi_{1}^{*}$ under $\mathrm{Q}^{w *}$. Using the fact that $\sum_{k=1}^{n} w_{k}=1$ and changing measures, we obtain

$$
\begin{aligned}
& \mathrm{E}(\langle u, \eta\rangle)_{+}=c \mathrm{E}_{\mathrm{Q}^{w}}\left(\left\langle u, \mathrm{e}^{\xi} \mathrm{e}^{-\xi_{1}}\right\rangle_{+} \mathrm{e}^{\xi_{1}} \mathrm{e}^{-\langle w, \xi\rangle}\right)=c \mathrm{E}_{\mathrm{Q}^{w}}\left(\left\langle u, \mathrm{e}^{\xi-1 \xi_{1}}\right\rangle_{+} \mathrm{e}^{-\left\langle w, \xi-1 \xi_{1}\right\rangle}\right), \\
& \left.\mathrm{E}\left(\left\langle u, \eta^{*}\right\rangle\right)_{+}=c \mathrm{E}_{\mathrm{Q}^{w *}}\left(\left\langle u, \mathrm{e}^{\xi^{*}-1} \xi_{1}^{*}\right\rangle_{+} \mathrm{e}^{-\left\langle w, \xi^{*}-1\right.} \xi_{1}^{*}\right\rangle\right) .
\end{aligned}
$$

Since $\xi-\mathbf{1} \xi_{1}$ under $\mathrm{Q}^{w}$ shares the distribution with $\xi^{*}-\mathbf{1} \xi_{1}^{*}$ under $\mathrm{Q}^{w *}$, the right-hand and, thus, also the left-hand sides coincide, i.e. we arrive at (3.2). It follows from the necessity that (3.3) holds for all $w$ such that the characteristic function is finite and $\sum w_{k}=1$.

Remark 3.2. By the generalised Hölder inequality, it follows from the integrability of $\eta$ and $\eta^{*}$ in Theorem 3.2 that the characteristic functions in (3.3) are finite for all $w$ from the unit simplex

$$
\Delta=\left\{x=\left(x_{1}, \ldots, x_{n}\right): x_{i} \geq 0, i=1, \ldots, n, \sum x_{i}=1\right\} .
$$

Thus, the set of all $w \in \mathbb{R}^{n}$ such that $\mathrm{e}^{\langle w, \xi\rangle}$ is integrable contains the unit simplex $\Delta$ if $\mathrm{e}^{\xi}$ is integrable itself.

Let $\mathscr{H}_{\beta}$ denote the family of nonnegative, positive $\beta$ homogeneous functions $g:(0, \infty)^{n} \mapsto$ $\mathbb{R}_{+}$, i.e. $g(c x)=c^{\beta} g(x)$ for all $c>0$ and $x \in(0, \infty)^{n}$. Note that $f_{\mathrm{b}}^{\mathrm{o}} \in \mathscr{H}_{1}$. Other examples of payoff functions of class $\mathscr{H}_{\beta}$ can be found in the literature about the duality principle; see, e.g. [18] and [23]. The following result says that the equality of expected payoffs from exchange options implies the equality of expected payoffs from the whole family $\mathscr{H}_{1}$, despite the fact that the asset prices do not necessarily coincide in distribution.

Theorem 3.3. If integrable random vectors $\eta=\mathrm{e}^{\xi}$ and $\eta^{*}=\mathrm{e}^{\xi^{*}}$ satisfy (3.2) (i.e. share the same zonoid), then $\mathrm{E} g(\eta)=\mathrm{E} g\left(\eta^{*}\right)$ for all $g \in \mathscr{H}_{1}$.

Proof. By choosing $u=e_{1}$ in (3.2) we arrive at $\mathrm{E} \eta_{1}=\mathrm{E} \eta_{1}^{*}$. Hence, (3.2) is equivalent to

$$
\mathrm{E}_{\mathrm{Q}^{1}}\left(u_{1}+\sum_{i=2}^{n} u_{i} \frac{\eta_{i}}{\eta_{1}}\right)_{+}=\mathrm{E}_{\mathrm{Q}^{1 *}}\left(u_{1}+\sum_{i=2}^{n} u_{i} \frac{\eta_{i}^{*}}{\eta_{1}^{*}}\right)_{+}
$$

for all $u \in \mathbb{R}^{n}$. By Theorem 1.1, the distribution of $\tilde{\kappa}_{1}(\eta)$ under $\mathrm{Q}^{1}$ coincides with the distribution of $\tilde{\kappa}_{1}\left(\eta^{*}\right)$ under $\mathrm{Q}^{1 *}$ so that

$$
\mathrm{E} g(\eta)=c \mathrm{E}_{\mathrm{Q}^{1}} g\left(\left(1, \tilde{\kappa}_{1}(\eta)\right)\right)=c \mathrm{E}_{\mathrm{Q}^{1 *}} g\left(\left(1, \tilde{\kappa}_{1}\left(\eta^{*}\right)\right)\right)=\mathrm{E} g\left(\eta^{*}\right)
$$

for all $g \in \mathscr{H}_{1}$.

It is possible to generalise this characterisation for functions from the family $\mathscr{H}_{\beta}$.

Theorem 3.4. Assume that random vectors $\eta=\mathrm{e}^{\beta \xi}$ and $\eta^{*}=\mathrm{e}^{\beta \xi^{*}}$ are integrable for some $\beta \in \mathbb{R}$. Then $\mathrm{E} g(\eta)=\mathrm{E} g\left(\eta^{*}\right)$ for all $g \in \mathscr{H}_{\beta}$ if and only if

$$
\varphi_{\xi}(u-\mathrm{i} w)=\varphi_{\xi}(u-\mathrm{i} w)
$$

for all $u \in \mathbb{H}$ and at least one (and then necessarily for all) $w \in \mathbb{R}^{n}$ such that $\sum w_{k}=\beta$ and the characteristic functions in (3.4) exist. 
Proof. Assume first that $\beta \neq 0$. If $g \in \mathcal{H}_{\beta}$ with $\beta \neq 0$, then $g_{1}(x)=g\left(x^{1 / \beta}\right)$, with the power operation applied coordinatewise being 1-homogeneous, and Theorem 3.3 applies. Thus, $\mathrm{E}(g(\eta))=\mathrm{E}\left(g\left(\eta^{*}\right)\right)$ for all $g \in \mathscr{H}_{\beta}$ if and only if (3.3) holds for the characteristic functions of $\beta \xi$ and $\beta \xi^{*}$ (corresponding to $\eta^{\beta}$ and $\left(\eta^{*}\right)^{\beta}$ ) for all $u \in \mathbb{H}$ and at least one (and then necessarily for all) $w$ with $\sum w_{k}=1$. Rewriting (3.3) for the characteristic functions of $\xi$ and $\xi^{*}$ yields (3.4).

Now let $\beta=0$. For all $u \in \mathbb{R}_{+}^{n}$, consider the integrable functions $g(\eta)=\left(u_{1}-\right.$ $\left.\sum_{i=2}^{n} u_{i} \eta_{i} / \eta_{1}\right)_{+} \in \mathcal{H}_{0}$. It follows from a version of Theorem 1.1 for positive random vectors from [25, Theorem 1.1] that $\tilde{\kappa}_{1}(\eta)$ and $\tilde{\kappa}_{1}\left(\eta^{*}\right)$ are identically distributed. Calculating the characteristic functions of $\xi$ and $\xi^{*}$ with an arbitrary complex argument $w$ yields (3.4) with $\sum w_{k}=0$ and the characteristic functions exist (at least) for all $w$ with vanishing imaginary part.

In the other direction, (3.4) implies that $\tilde{\kappa}_{1}(\eta)$ under $\mathrm{Q}^{w}$ and $\tilde{\kappa}_{1}\left(\eta^{*}\right)$ under $\mathrm{Q}^{w *}$ are identically distributed. Here we have also used the fact that (3.4) yields $\mathrm{Ee}^{\langle w, \xi\rangle}=\mathrm{Ee}^{\left\langle w, \xi^{*}\right\rangle}=c$. Let $g \in \mathscr{H}_{0}$. Then $\mathrm{e}^{-\langle w, \xi\rangle}=\mathrm{e}^{-\left\langle w, \xi-1 \xi_{1}\right\rangle}$ is a function of $\left(1, \tilde{\kappa}_{1}(\eta)\right)$, so that we can define $f\left(\tilde{\kappa}_{1}(\eta)\right)=g\left(\left(1, \tilde{\kappa}_{1}(\eta)\right)\right) \mathrm{e}^{-\left\langle w, \xi-1 \xi_{1}\right\rangle}$. Then

$$
\mathrm{E} g(\eta)=c \mathrm{E}_{\mathrm{Q}^{w}} f\left(\tilde{\kappa}_{1}(\eta)\right)=c \mathrm{E}_{\mathrm{Q}^{w *}} f\left(\tilde{\kappa}_{1}\left(\eta^{*}\right)\right)=\mathrm{E} g\left(\eta^{*}\right) .
$$

Corollary 3.1 below follows directly from Theorem 3.2 upon noting that the $i j$-swapinvariance of $\eta$ means that $\eta$ and $\pi_{i j}(\eta)$ have equal zonoids, or, equivalently, that

$$
\mathrm{E}(\langle u, \eta\rangle)_{+}=\mathrm{E}\left(\left\langle\pi_{i j}(u), \eta\right\rangle\right)_{+}=\mathrm{E}\left(\left\langle u, \pi_{i j}(\eta)\right\rangle\right)_{+} \quad \text { for all } u \in \mathbb{R}^{n} .
$$

Corollary 3.1. An integrable random vector $\eta=\mathrm{e}^{\xi}$ is ij-swap-invariant if and only if the characteristic function of $\xi$ satisfies

$$
\varphi_{\xi}(u-\mathrm{i} w)=\varphi_{\xi}\left(\pi_{i j}(u-\mathrm{i} w)\right)
$$

for all $u \in \mathbb{H}$ and at least one (and then necessarily for all) $w \in \Delta$.

While the characteristic function (3.5) exists for all $w \in \Delta$, it is possible to relax the latter condition. Namely, integrable $\eta$ is $i j$-swap-invariant if and only if (3.5) holds for all $u \in \mathbb{H}$ and at least one (and then necessarily for all) $w$ such that $\sum_{k=1}^{n} w_{k}=1$ and one side of (3.5) is finite, in other words such that $\mathrm{Ee}^{\langle w, \xi\rangle}<\infty$.

The complex shifts on both sides of (3.5) are the same if $w_{i}=w_{j}$. In the most important special case $w=\frac{1}{2} e_{i j}$ with $e_{i j}=e_{i}+e_{j}$, so that the $i j$-swap-invariance characterisation reads

$$
\varphi_{\xi}\left(u-\mathrm{i} \frac{1}{2} e_{i j}\right)=\varphi_{\xi}\left(\pi_{i j}(u)-\mathrm{i} \frac{1}{2} e_{i j}\right), \quad u \in \mathbb{H} .
$$

Corollary 3.2. An integrable random vector $\eta=\mathrm{e}^{\xi}$ is ij-swap-invariant if and only if the orthogonal projection of $\xi$ onto $\mathbb{H}$ is i j-exchangeable under the probability measure $\mathrm{Q}^{w}$ for at least one (and then necessarily for all) $w \in \Delta$ such that $w_{i}=w_{j}$.

Remark 3.3. (Independency and self-duality in the bivariate case.) Consider a bivariate integrable random vector $\eta=\left(\eta_{1}, \eta_{2}\right)$ with independent components. A sufficient condition for $\eta$ to be swap invariant is that both $\eta_{1}$ and $\eta_{2}$ are self-dual random variables, since then we have, for arbitrary $\left(u_{1}, u_{2}\right) \in \mathbb{R}^{2}$,

$$
\begin{aligned}
\mathrm{E}\left(u_{1} \eta_{1}+u_{2} \eta_{2}\right)_{+} & =\mathrm{E}\left(\mathrm{E}\left(\left(u_{1} \eta_{1}+u_{2} \eta_{2}\right)_{+} \mid \eta_{1}\right)\right) \\
& =\mathrm{E}\left(\mathrm{E}\left(\left(u_{1} \eta_{1} \eta_{2}+u_{2}\right)_{+} \mid \eta_{2}\right)\right) \\
& =\mathrm{E}\left(u_{1} \eta_{2}+u_{2} \eta_{1}\right)_{+} .
\end{aligned}
$$


Note that this construction does not apply for $\eta$ of dimension 3 and more. Nonexchangeable swap-invariant random vectors with independent, not necessarily self-dual components can be constructed in the following way. Consider integrable, independent, and identically distributed $\zeta_{1}, \zeta_{2}$ and self-dual $\tilde{\zeta}_{1}, \tilde{\zeta}_{2}$ all jointly independent. It is easy to see that $\left(\eta_{1}, \eta_{2}\right)=\left(\zeta_{1} \tilde{\zeta}_{1}, \zeta_{2} \tilde{\zeta}_{2}\right)$ is a swap-invariant random vector with not necessarily self-dual independent components. It is apparent from [30] that the product of a self-dual random variable and a general random variable is not necessarily self-dual.

\section{Weighted swap invariance}

The introduced swap-invariance concept relies on invariance properties of the payoff function $f_{\mathrm{b}}^{\mathrm{o}}$ from (1.2). It is also possible to modify this payoff function by introducing a positive weight given by a random variable $\mathrm{e}^{\vartheta}$. A random vector $\eta$ is called weighted $i j$-swap-invariant if $\mathrm{e}^{\vartheta} \eta$ is integrable and

$$
\mathrm{E}\left(\mathrm{e}^{\vartheta} f_{\mathrm{b}}^{\mathrm{o}}(u)\right)=\mathrm{E}\left(\mathrm{e}^{\vartheta} f_{\mathrm{b}}^{\mathrm{o}}\left(\pi_{i j}(u)\right)\right) \quad \text { for all } u \in \mathbb{R}^{n} .
$$

In this case we write $\eta \in \mathrm{WS}_{i j}(\vartheta)$. The involved payoff function is typical for so-called quanto-swap options.

Theorem 4.1. Let $\eta=\mathrm{e}^{\xi}$ be a random vector, and let $\mathrm{e}^{\vartheta}$ be a random variable such that $\mathrm{e}^{\vartheta} \eta$ is integrable. Then $\eta \in \mathrm{WS}_{i j}(\vartheta)$ if and only if

$$
\varphi_{\xi+1} \vartheta(u-\mathrm{i} w)=\varphi_{\xi+1} \vartheta\left(\pi_{i j}(u-\mathrm{i} w)\right)
$$

for all $u \in \mathbb{H}$ and at least one (and then necessarily for all) $w \in \Delta$.

Proof. It suffices to note that $\mathrm{e}^{\vartheta} \eta$ and $\mathrm{e}^{\vartheta} \pi_{i j}(\eta)$ share the same zonoid and apply Theorem 3.2.

If $w=\frac{1}{2} e_{i j}$ then (4.2) simplifies to

$$
\varphi_{\xi+\mathbf{1} \vartheta}\left(u-\mathrm{i} \frac{1}{2} e_{i j}\right)=\varphi_{\xi+\mathbf{1}} \vartheta\left(\pi_{i j}(u)-\mathrm{i} \frac{1}{2} e_{i j}\right) \quad \text { for all } u \in \mathbb{H} .
$$

If the log-weight $\vartheta$ is given by a linear combination of the log-prices of the assets included in $f_{\mathrm{b}}^{\mathrm{o}}(u)$, i.e. $\vartheta=\langle\xi, v\rangle$ for some $v \in \mathbb{R}^{n}$, we obtain the following result.

Corollary 4.1. Let $\eta=\mathrm{e}^{\xi}$ be a random vector such that $\mathrm{e}^{\vartheta} \eta$ is integrable with $\vartheta=\langle v, \xi\rangle$ for some $v \in \mathbb{R}^{n}$. Then $\eta \in \mathrm{WS}_{i j}(\vartheta)$ if and only if

$$
\varphi_{\xi}(u-\mathrm{i} w-\mathrm{i} v)=\varphi_{\xi}\left(\pi_{i j}(u-\mathrm{i} w)-\mathrm{i} v\right)
$$

for all $u \in \mathbb{H}$ and at least one (and then necessarily for all) $w \in \Delta$.

Proof. Since $u \in \mathbb{H}$ and $w \in \Delta$,

$$
\begin{aligned}
\varphi_{\xi+1} \vartheta(u-\mathrm{i} w) & =\mathrm{Ee}^{\mathrm{i}(\langle u-\mathrm{i} w, \xi+\mathbf{1}\langle v, \xi\rangle\rangle)} \\
& =\mathrm{Ee}^{\mathrm{i}\langle u-\mathrm{i} w, \xi\rangle+\mathrm{i}\langle v, \xi\rangle(\langle u, \mathbf{1}\rangle-\mathrm{i}\langle w, \mathbf{1}\rangle)} \\
& =\mathrm{Ee}^{\mathrm{i}\langle u-\mathrm{i} w-\mathrm{i} v, \xi\rangle}
\end{aligned}
$$

and analogously $\varphi_{\xi+1} \vartheta\left(\pi_{i j}(u-\mathrm{i} w)\right)=\varphi_{\xi}\left(\pi_{i j}(u-\mathrm{i} w)-\mathrm{i} v\right)$. 


\section{Swap invariance for Lévy models}

In this section we assume that $\eta=\mathrm{e}^{\xi}$ with $\xi$ being infinitely divisible, i.e. $\xi=L_{1}$ for a Lévy process $L_{t}, t \geq 0$; see [35]. In order to handle possibly weighted cases, consider also a random variable $\zeta$ such that $(\xi, \zeta)$ is infinitely divisible.

Define the linear transformation (actually orthogonal projection) which maps every $x \in$ $\mathbb{R}^{n+1}$ onto the hyperplane $\mathbb{H}$ in the space of dimension $n$ acting as $P x$ with the matrix

$$
P=\left(\begin{array}{ccccc}
1-\frac{1}{n} & -\frac{1}{n} & \cdots & -\frac{1}{n} & 0 \\
-\frac{1}{n} & 1-\frac{1}{n} & \cdots & -\frac{1}{n} & 0 \\
\vdots & \vdots & \vdots & \vdots & \vdots \\
-\frac{1}{n} & -\frac{1}{n} & \cdots & 1-\frac{1}{n} & 0
\end{array}\right)
$$

Corollary 3.1, Theorem 4.1, and Corollary 4.1 provide many equivalent characterisations of the (weighted) $i j$-swap-invariance in terms of various $w \in \Delta \subset \mathbb{R}^{n}$. In order to simplify the calculations, we let $w=\frac{1}{2} e_{i j}=\frac{1}{2}\left(e_{i}+e_{j}\right)$ in the sequel (so that $w$ is $\pi_{i j}$-invariant), and we can consider $e_{i}$ and $e_{j}$ to be standard basis vectors in $\mathbb{R}^{n+1}$. Sometimes we add the zero component to the vectors $u \in \mathbb{H}$ and then write $(u, 0)$.

Theorem 5.1. Let $(\xi, \zeta)$ be an infinitely divisible $(n+1)$-dimensional random vector such that $\mathrm{e}^{\langle v,(\xi, \zeta)\rangle} \mathrm{e}^{\xi}$ is integrable for some $v \in \mathbb{R}^{n+1}$. Then $\eta=\mathrm{e}^{\xi} \in \mathrm{WS}_{i j}(\langle v,(\xi, \zeta)\rangle)$ if and only if the characteristic triplet $(A, v, \gamma)$ of $(\xi, \zeta)$ satisfies the following conditions.

(a) If $n \geq 3$, the matrix $A$ satisfies

$$
a_{l i}-a_{l j}=\frac{1}{2}\left(a_{i i}-a_{j j}\right)
$$

for all $l \neq i, j, l \leq n$.

(b) The image $\hat{v} P^{-1}$ under $P$ of measure

$$
\mathrm{d} \hat{v}(x)=\mathrm{e}^{\left\langle\mathrm{e}_{i j} / 2+v, x\right\rangle} \mathrm{d} v(x), \quad x \in \mathbb{R}^{n+1},
$$

is $\pi_{i j}$-invariant on $\mathbb{H} \backslash\{0\}$.

(c) $\gamma$ satisfies

$$
\begin{aligned}
\gamma_{i}-\gamma_{j}= & \frac{1}{2}\left(a_{j j}-a_{i i}\right)+\sum_{k=1}^{n+1}\left(a_{j k}-a_{i k}\right) v_{k} \\
& +\int_{\mathbb{R}^{n+1}}\left(x_{j}-x_{i}\right)\left(\mathrm{e}^{\left\langle\mathrm{e}_{i j} / 2+v, x\right\rangle} 1_{\{\|P x\| \leq 1\}}-1_{\{\|x\| \leq 1\}}\right) \mathrm{d} v(x) .
\end{aligned}
$$

Proof. Since $u \in \mathbb{H}$, we can express (4.3) in terms of the joint characteristic function of $(\xi, \zeta)$, i.e.

$$
\varphi_{(\xi, \zeta)}\left((u, 0)-\mathrm{i}\left(\frac{1}{2} e_{i j}+v\right)\right)=\varphi_{(\xi, \zeta)}\left(\pi_{i j}(u, 0)-\mathrm{i}\left(\frac{1}{2} e_{i j}+v\right)\right),
$$

where from now on $\pi_{i j}$ stands for the corresponding permutation matrix of appropriate dimension. Let $\hat{\mathrm{Q}}$ be the Esscher transform (3.1) of $\mathrm{Q}$ with parameter $w=\frac{1}{2} e_{i j}+v$. The characteristic 
triplet $(\hat{A}, \hat{v}, \hat{\gamma})$ of $(\xi, \zeta)$ under $\hat{Q}$ is given by $\hat{A}=A$, the new Lévy measure $\hat{v}$ is given by (5.2), and

$$
\hat{\gamma}=\gamma+A\left(\frac{1}{2} e_{i j}+v\right)+\int_{\mathbb{R}^{n+1}} x\left(\mathrm{e}^{\left\langle e_{i j} / 2+v, x\right\rangle}-1\right) 1_{\{\|x\| \leq 1\}} \mathrm{d} v(x)
$$

see [36, Example 7.3] and [35, Theorem 25.17] for the extension of the Lévy-Khintchine formula to the needed subset in the $(n+1)$-dimensional complex plane in view of the imposed integrability conditions.

By (5.5) and in view of Corollary 3.2, the weighted swap invariance of $\eta$ means that $(\xi, \zeta)$ projected by $P$ onto $\mathbb{H}$ is $i j$-exchangeable under $\hat{\mathrm{Q}}$. This projection has the characteristic triplet $\left(A^{\prime}, v^{\prime}, \gamma^{\prime}\right)$, where $A^{\prime}=P \hat{A} P^{\top}=P A P^{\top}, v^{\prime}=\hat{v} P^{-1}$ is the projection of $\hat{v}$ on $\mathbb{H} \backslash\{0\}$, and

$$
\gamma^{\prime}=P \hat{\gamma}+\int_{\mathbb{R}^{n+1}} P x\left(1_{\{\|P x\| \leq 1\}}-1_{\{\|x\| \leq 1\}}\right) \mathrm{d} \hat{v}(x) ;
$$

see [35, Proposition 11.10]. The elements of $A^{\prime}$ can be calculated as

$$
a_{i j}^{\prime}=a_{i j}-\frac{1}{n}\left(\sum_{k=1}^{n} a_{k i}+\sum_{k=1}^{n} a_{k j}\right)+\frac{1}{n^{2}} \sum_{k, l=1}^{n} a_{l k} .
$$

Since the projection of $(\xi, \zeta)$ is $i j$-exchangeable, Proposition 2.1(a) requires $a_{i i}^{\prime}=a_{j j}^{\prime}$, so that

$$
a_{i i}-\frac{2}{n} \sum_{k=1}^{n} a_{i k}=a_{j j}-\frac{2}{n} \sum_{k=1}^{n} a_{j k} .
$$

Furthermore, $a_{l i}^{\prime}=a_{l j}^{\prime}$ for $l \neq i, j$ yields (5.1), which also always satisfies (5.8). By Proposition $2.1, v^{\prime}$ is symmetric with respect to $\pi_{i j}$ and $\gamma_{i}^{\prime}=\gamma_{j}^{\prime}$. By combining (5.7) with (5.6) we obtain (5.3).

By combining Theorem 5.1 with [35, Proposition 11.10] and changing variables, or adapting the proof of Theorem 5.1, we obtain the following result.

Corollary 5.1. The integrable random vector $\eta=\mathrm{e}^{\xi}$ with infinitely divisible $\xi$ having the Lévy triplet $(A, v, \gamma)$ is ij-swap-invariant if and only if Theorem 5.1(a) holds for the $n \times n$ matrix $A$, the orthogonal projection of measure

$$
\mathrm{d} \bar{v}(x)=\mathrm{e}^{\left(x_{i}+x_{j}\right) / 2} \mathrm{~d} \nu(x), \quad x \in \mathbb{R}^{n},
$$

on $\mathbb{H} \backslash\{0\}$ is $\pi_{i j}$-invariant, and

$$
\gamma_{i}-\gamma_{j}=\frac{1}{2}\left(a_{j j}-a_{i i}\right)+\int_{\mathbb{R}^{n}}\left(x_{j}-x_{i}\right)\left(\mathrm{e}^{\left(x_{i}+x_{j}\right) / 2} 1_{\left\{\left\|P^{\prime} x\right\| \leq 1\right\}}-1_{\{\|x\| \leq 1\}}\right) \mathrm{d} v(x),
$$

where $P^{\prime}$ is $P$ with the last column omitted.

The following theorem shows that the condition on the drift $\gamma$ from the Lévy triplet is automatically satisfied in the case of equal means.

Theorem 5.2. Let $\eta=\mathrm{e}^{\xi}$ be an $n$-dimensional integrable random vector with infinitely divisible $\xi$ and such that $\mathrm{E} \eta_{i}=\mathrm{E} \eta_{j}$. Then $\eta$ is $i j$-swap-invariant if and only if the characteristic triplet $(A, v, \gamma)$ of $\xi$ satisfies the first two conditions of Corollary 5.1 (i.e. (5.10) always holds in this case). 
Proof. Since $\mathrm{E} \eta_{l}=\varphi_{\xi}^{\mathrm{Q}}\left(-\mathrm{i} e_{l}\right)$ for $l=i, j$ (where here $\left.e_{l} \in \mathbb{R}^{n}\right)$,

$$
\begin{aligned}
\gamma_{i}+ & \frac{1}{2} a_{i i}+\int_{\mathbb{R}^{n}}\left(\mathrm{e}^{x_{i}}-1-x_{i} 1_{\{\|x\| \leq 1\}}\right) \mathrm{d} \nu(x) \\
& =\gamma_{j}+\frac{1}{2} a_{j j}+\int_{\mathbb{R}^{n}}\left(\mathrm{e}^{x_{j}}-1-x_{j} 1_{\{\|x\| \leq 1\}}\right) \mathrm{d} \nu(x) .
\end{aligned}
$$

In this case (5.10) becomes

$$
\int_{\mathbb{R}^{n}}\left(\mathrm{e}^{\left(x_{i}-x_{j}\right) / 2}-\mathrm{e}^{\left(x_{j}-x_{i}\right) / 2}+\left(x_{j}-x_{i}\right) 1_{\left\{\left\|P^{\prime} x\right\| \leq 1\right\}}\right) \mathrm{d} \bar{v}(x)=0 .
$$

Making the change of variable $x=x^{\prime}+x^{\prime \prime}$ with $x^{\prime} \in \mathbb{H}$ and $x^{\prime \prime} \in \mathbb{H}^{\perp}$ and noting that $\mathbb{H}^{\perp}=\{t \mathbf{1}: t \in \mathbb{R}\}$ consists of vectors with all equal components, the integral becomes an integral over $\mathbb{H}$ with respect to the projection of $\bar{v}$ onto $\mathbb{H}$. The integrand changes the sign if $x$ is replaced by $\pi_{i j}(x)$, while the projected measure $\bar{v}$ is invariant on $\mathbb{H} \backslash\{0\}$ (where the integrand is nonvanishing) under this change. Thus, the whole integral vanishes.

Remark 5.1. (Risk-neutral nonweighted case.) It is worth noting that the assumption $\mathrm{E} \eta_{i}=$ $\mathrm{E} \eta_{j}$ in Theorem 5.2 is satisfied in a risk-neutral setting, where $\mathrm{E} \eta_{l}=1, l=1, \ldots, n$.

Example 5.1. (Two-asset case.) In the bivariate nonweighted infinitely divisible (Lévy) case the first condition of Corollary 5.1 is vacuous. The second condition holds, e.g. for exchangeable $v$, while the third condition always holds in the risk-neutral setting.

Example 5.2. (Log-normal distribution.) If the Lévy measure vanishes, the first condition of Theorem 5.1 remains the same, the second condition always holds, while the third condition becomes (with $\mu$ replacing $\gamma$ )

$$
\mu_{i}-\mu_{j}=\frac{1}{2}\left(a_{j j}-a_{i i}\right)+\sum_{k=1}^{n+1}\left(a_{j k}-a_{i k}\right) v_{k} .
$$

Under a risk-neutral assumption, this condition means that $\sum_{k=1}^{n+1}\left(a_{j k}-a_{i k}\right) v_{k}=0$, so in the nonweighted risk-neutral setting only the first condition of Corollary 5.1 is imposed.

In particular, each bivariate risk-neutral log-normal distribution is (nonweighted) swap invariant, no matter what the volatilities of the assets and correlation are. In the nonweighted risk-neutral setting with $n=3$ and $i=1, j=2$ the only condition is

$$
a_{31}-a_{32}=\frac{1}{2}\left(a_{11}-a_{22}\right) .
$$

In the presence of a weight $\left(\xi_{1}, \xi_{2}, \zeta\right)$, i.e. $\vartheta=\zeta$, in the risk-neutral case the only condition $a_{13}=a_{23}$ on the covariance matrix of $\left(\xi_{1}, \xi_{2}, \zeta\right)$ guarantees the weighted swap-invariance property

$$
\mathrm{Ee}^{\zeta}\left(u_{1} \eta_{1}+u_{2} \eta_{2}\right)_{+}=\mathrm{Ee}^{\zeta}\left(u_{2} \eta_{1}+u_{1} \eta_{2}\right)_{+}, \quad\left(u_{1}, u_{2}\right) \in \mathbb{R}^{2} .
$$

If the weight is determined by the prices of the assets included in the swap, namely $\vartheta=\langle v, \xi\rangle$, the swap-invariance condition reads $v_{1} a_{11}-v_{2} a_{22}=\left(v_{1}-v_{2}\right) a_{12}$.

Consider a higher-dimensional risk-neutral log-normal setting with the weight $\vartheta=\langle v, \xi\rangle$ determined by the assets included in $f_{\mathrm{b}}^{\mathrm{o}}(u)$ in a rather general way with $v \notin \mathbb{H}$ and $v_{i}=v_{j}$ (in particular, $v=e_{k}$ with $\left.k \neq i, j\right)$. Then $\eta \in \mathrm{WS}_{i j}(\langle v, \xi\rangle)$ implies the $i j$-exchangeability of $\eta$. Indeed, the risk neutrality reduces (5.3) to $\sum_{k=1}^{n}\left(a_{j k}-a_{i k}\right) v_{k}=0$. By $v_{i}=v_{j}$ and (5.1), this yields $\frac{1}{2}\left(a_{i i}-a_{j j}\right)\langle\mathbf{1}, v\rangle=0$, whence $a_{i i}=a_{j j}$ by $v \notin \mathbb{H}$. Taking into account (5.1), we also have $a_{l i}=a_{l j}$ for all $l \neq i, j$, so that the exchangeability follows from Proposition 2.1. 
Remark 5.2. (Square integrable case and covariance.) Theorem 5.1(a) yields a certain restriction on the correlation structure arising from the centred Gaussian term for $n \geq 3$, while, for $n=2$, there are no restrictions. In order to also relax the restrictions for higher-dimensional models, it is useful to introduce a jump component. Assume that $\int_{\|x\|>1}\|x\|^{2} \mathrm{~d} v(x)<\infty$, i.e. $(\xi, \zeta)$ is square integrable. Then the covariance matrix of $(\xi, \zeta)$ has elements

$$
\Sigma_{l j}=\left(a_{l j}+\int x_{l} x_{j} \mathrm{~d} v(x)\right), \quad l, j=1, \ldots, n+1 ;
$$

see [35, Example 25.12]. Thus, despite some constraints on the Lévy measure given in Theorem 5.1 and Corollary 5.1, there is more flexibility in modelling the correlation structure of $\eta$.

Remark 5.3. (Lévy measures based on exchangeability.) The image of the Lévy measure $\hat{v}$ under $P$ is $\pi_{i j}$-invariant if (but not only if) the Lévy measure $\hat{v}$ is $\pi_{i j}$-invariant itself. A simple example of Lévy measures satisfying Theorem 5.1(b) can be constructed by taking an $(n+1)$ dimensional $i j$-exchangeable (i.e. $\pi_{i j}$-invariant) Lévy measure $\hat{v}$ satisfying (2.1), and defining $v$ from (5.2) given that the imposed integrability assumption on $\mathrm{e}^{\langle v,(\xi, \zeta)\rangle} \eta$ is satisfied.

Example 5.3. (Compound Poisson distribution.) Assume that the Lévy measure is finite with existing first exponential moments. Without loss of generality, assume that its total mass is 1 . Then $\bar{v}$ from Corollary 5.1 is, up to a constant, the Esscher transform of $v$ with parameter $\frac{1}{2} e_{i j}$. Thus, the invariance of its projection onto $\mathbb{H}$ is equivalent to

$$
\varphi_{\nu}\left(u-\mathrm{i} \frac{1}{2} e_{i j}\right)=\varphi_{\nu}\left(\pi_{i j}(u)-\mathrm{i} \frac{1}{2} e_{i j}\right), \quad u \in \mathbb{H},
$$

for the characteristic function of $v$, which exactly corresponds to (3.6). Hence, the distribution of the logarithm of any $i j$-swap-invariant vector $\eta$ can be chosen to serve as the Lévy measure $v$ (where $v(\{0\})$ is set to 0 if $\eta$ has an atom at $(1, \ldots, 1)$ ). For instance, Lévy measures satisfying (5.9) can be created from normal distributions described in Example 5.2. In the bivariate case this imposes only a slight restriction on the expectations, while the variances and correlation are not restricted.

Example 5.4. (Swap invariance in bivariate generalized hyperbolic models.) Consider a riskneutral bivariate generalised hyperbolic case, i.e. $\eta=\mathrm{e}^{\xi}$, where $\left(\xi_{1}, \xi_{2}\right) \sim \mathrm{GH}_{2}(\lambda, \alpha, \beta, \delta, \mu, \Delta)$ (cf. [5]), with corresponding parameters $\lambda \in \mathbb{R}, \alpha, \delta \in \mathbb{R}_{+}, \mu, \beta \in \mathbb{R}^{2}$, and $\Delta$ a symmetric, positive definite, $2 \times 2$ matrix, where without loss of generality $\operatorname{det}(\Delta)=1$. Following [18, Example 5.9] based on [29], assume that $\delta>0$ and $\alpha^{2}-\langle\beta, \Delta \beta\rangle>0$ so that the moments of all orders exist and the Lévy measure $v$ has a density $v(x)$ given by

$$
\begin{aligned}
v(x)=\frac{\mathrm{e}^{\langle\beta, x\rangle}}{\pi \sqrt{\left\langle x, \Delta^{-1} x\right\rangle}}( & \int_{0}^{\infty} \frac{\sqrt{2 y+\alpha^{2}} K_{1}\left(\sqrt{\left(2 y+\alpha^{2}\right)\left\langle x, \Delta^{-1} x\right\rangle}\right)}{\pi^{2} y\left(J_{|\lambda|}^{2}(\delta \sqrt{2 y})+Y_{|\lambda|}^{2}(\delta \sqrt{2 y})\right)} \mathrm{d} y \\
& \left.+\alpha K_{1}\left(\alpha \sqrt{\left\langle x, \Delta^{-1} x\right\rangle}\right) \lambda 1_{\{\lambda>0\}}\right)
\end{aligned}
$$

where $J_{\iota}, Y_{\iota}$, and $K_{\iota}$ denote the (modified) Bessel functions of the first, second, and third kinds with index $\iota$, and where further conditions on the parameters for ensuring the existence of the exponential moments can immediately be obtained from [40, Remark 2.2].

The parameters for $\tilde{\xi}=\xi_{2}-\xi_{1}$ under $\mathrm{Q}^{1}$ are calculated in [18, Example 5.9], in particular

$$
\tilde{\beta}=s \frac{\beta_{2} \delta_{22}-\left(\beta_{1}+1\right) \delta_{11}-\delta_{12}\left(\beta_{2}-\beta_{1}-1\right)}{\left(\delta_{11}+\delta_{22}-2 \delta_{12}\right)} .
$$


By Theorem 3.1 or Example 3.1, $\left(\mathrm{e}^{\xi_{1}}, \mathrm{e}^{\xi_{2}}\right)$ is swap invariant if and only if $\mathrm{e}^{\tilde{\xi}}$ is self-dual under $\mathrm{Q}^{1}$. However, following [22] in the risk-neutral setting, this is the case if and only if $\tilde{\beta}=-\frac{1}{2}$, so we obtain the slight restriction

$$
2\left(\delta_{22}-\delta_{12}\right) \beta_{2}+\delta_{22}=2\left(\delta_{11}-\delta_{12}\right) \beta_{1}+\delta_{11} .
$$

Hence, the considerable effective degrees of freedom for modelling two assets based on the considered dependent generalised hyperbolic Lévy processes only slightly diminish by additionally imposing the bivariate swap-invariance property.

By interpreting $(\xi, \zeta)$ as the time-one value of a Lévy process, we arrive at the following result.

Corollary 5.2. If $\left(\xi_{t}, \zeta_{t}\right), t \geq 0$, is the Lévy process that satisfies the conditions of Theorem 5.1 then $\mathrm{e}^{\xi_{t}}$ is weighted $i j$-swap-invariant for all $t \geq 0$.

Remark 5.4. (Random times.) Consider a family $\{\eta(t), t \geq 0\}$ of $i j$-swap-invariant random vectors. Let $\tau_{t}, t \geq 0$, be an increasing nonnegative random function independent of $\eta$. If the time-changed stochastic process $\eta\left(\tau_{t}\right), t \geq 0$, is integrable for all $t$ then $\eta\left(\tau_{t}\right)$ is also $i j$-swap-invariant.

\section{Quasi-swap-invariance}

In some cases the swap-invariance condition is too restrictive; in particular, its adjusted variant is useful to take into account unequal carrying costs. We say that $\eta$ is quasi-swapinvariant if

$$
\mathrm{E}\left(\mathrm{e}^{\vartheta} f_{\mathrm{b}}^{\mathrm{o}}(u)\right)=\mathrm{E}\left(\mathrm{e}^{\vartheta} f_{\mathrm{b}}^{\mathrm{o}}\left(\pi_{i j}(u)\right)\left(\frac{\eta_{i}}{\eta_{j}}\right)^{\alpha}\right)
$$

for all $u \in \mathbb{R}^{n}$ and all mentioned expectations exist. Note that this property is not symmetric with respect to $i$ and $j$.

By passing to the new probability measure $\tilde{\mathrm{Q}}^{j}$ defined by (3.1) with $w=e_{j}+e_{n+1}$ for $\vartheta=\zeta$ and $w=e_{j}+\langle v,(\xi, \zeta)\rangle$ for $\vartheta=\langle v,(\xi, \zeta)\rangle$, assuming the $\tilde{\mathrm{Q}}^{j}$-integrability of $\tilde{\kappa}_{j}(\eta)$ as well as $\tilde{\kappa}_{j}(\eta)^{\alpha+1}$, and using [30, Theorem 5.2] (with vanishing $\lambda$ ), it is easy to see that (6.1) with $\alpha \neq-1$ is equivalent to the fact that $\tilde{\kappa}_{j}(\eta)^{\alpha+1}$ is self-dual with respect to the $i$ th numeraire under $\tilde{\mathrm{Q}}^{j}$. Random vectors that become self-dual if normalised and raised to some power are called quasi-self-dual in [30].

Theorem 6.1. Let $\eta=\mathrm{e}^{\xi}$ be a random vector such that $\mathrm{e}^{\vartheta} \eta$ and $\mathrm{e}^{\vartheta}\left(\eta_{i} / \eta_{j}\right)^{\alpha} \eta$ are integrable. Then (6.1) holds if and only if

$$
\varphi_{\xi+1} \vartheta(u-\mathrm{i} w)=\varphi_{\xi+1} \vartheta\left(\pi_{i j}(u-\mathrm{i} w)-\mathrm{i} \alpha\left(e_{i}-e_{j}\right)\right)
$$

for all $u \in \mathbb{H}$ and at least one (and then necessarily for all) $w \in \Delta$.

Proof. Define $\vartheta^{\prime}=\vartheta+\alpha \xi_{i}-\alpha \xi_{j}$, and note that $\mathrm{e}^{\vartheta} \eta$ and $\mathrm{e}^{\vartheta^{\prime}} \eta$ are integrable. Then (6.1) means that $\mathrm{e}^{\vartheta} \eta$ and $\pi_{i j}\left(\mathrm{e}^{\vartheta^{\prime}} \eta\right)$ share the same zonoid. By Theorem 3.2, this holds if and only if

$$
\varphi_{\xi+1 \vartheta}(u-\mathrm{i} w)=\varphi_{\pi_{i j}\left(\xi+1 \vartheta^{\prime}\right)}(u-\mathrm{i} w)
$$

for all $u \in \mathbb{H}$ and at least one (and then necessarily for all) $w \in \Delta$. It remains to note that the right-hand side is

$$
\varphi_{\xi+1} \vartheta^{\prime}\left(\pi_{i j}(u-\mathrm{i} w)\right)=\varphi_{\xi+1} \vartheta\left(\pi_{i j}(u-\mathrm{i} w)-\mathrm{i} \alpha\left(e_{i}-e_{j}\right)\right) .
$$


Since $\mathrm{e}^{\vartheta} \eta$ and $\pi_{i j}\left(\mathrm{e}^{\vartheta^{\prime}} \eta\right)$ share the same zonoid, it follows from Theorem 3.3 that $(6.1)$ implies that

$$
\mathrm{E}\left(\mathrm{e}^{\vartheta} g(\eta)\right)=\mathrm{E}\left(\mathrm{e}^{\vartheta} g\left(\pi_{i j}(\eta)\right)\left(\frac{\eta_{i}}{\eta_{j}}\right)^{\alpha}\right)
$$

for all $g \in \mathscr{H}_{1}$.

The assumption that $w \in \Delta$ in Theorem 6.1 can be replaced by the assumption that $\sum w_{k}=1$ and at least one side of (6.2) is finite. Note that the integrability of $\mathrm{e}^{\vartheta} \eta$ and $\mathrm{e}^{\vartheta}\left(\eta_{i} / \eta_{j}\right)^{\alpha} \eta$ implies that $\mathrm{Ee}^{(1+\alpha) \xi_{i} / 2+(1-\alpha) \xi_{j} / 2+\vartheta}<\infty$. Hence, we can choose $w=\frac{1}{2}(1+\alpha) e_{i}+\frac{1}{2}(1-\alpha) e_{j}$ so that (6.2) becomes

$$
\varphi_{\xi+\mathbf{1} \vartheta}\left(u-\mathrm{i}\left[\frac{1+\alpha}{2} e_{i}+\frac{1-\alpha}{2} e_{j}\right]\right)=\varphi_{\xi+1} \vartheta\left(\pi_{i j}(u)-\mathrm{i}\left[\frac{1+\alpha}{2} e_{i}+\frac{1-\alpha}{2} e_{j}\right]\right)
$$

for all $u \in \mathbb{H}$, i.e. the complex shifts on both sides of (6.4) are identical. For $\vartheta=\langle v,(\xi, \zeta)\rangle$, we can use the fact that $u \in \mathbb{H}$ in order to express (6.4) in terms of the joint characteristic function of $(\xi, \zeta)$ as

$$
\varphi_{(\xi, \zeta)}\left((u, 0)-\mathrm{i}\left[\frac{1}{2} e_{i j}+\frac{1}{2} \alpha\left(e_{i}-e_{j}\right)+v\right]\right)=\varphi_{(\xi, \zeta)}\left(\pi_{i j}(u, 0)-\mathrm{i}\left[\frac{1}{2} e_{i j}+\frac{1}{2} \alpha\left(e_{i}-e_{j}\right)+v\right]\right)
$$

for all $u \in \mathbb{H}$. Hence, (6.5) corresponds to (5.5) written for

$$
v^{\prime}=v+\frac{\alpha}{2}\left(e_{i}-e_{j}\right)
$$

instead of $v$. Thus, in the infinite divisible case, under suitable integrability assumptions, the quasi-swap-invariance holds if and only if the conditions of Theorem 5.1 are satisfied with $v$ replaced by $v^{\prime}$ given above, so that we immediately obtain the following result.

Corollary 6.1. Let $\eta=\mathrm{e}^{\xi}$ be a random vector such that $\mathrm{e}^{\langle v,(\xi, \zeta)\rangle} \eta$ and $\mathrm{e}^{\langle v,(\xi, \zeta)\rangle}\left(\eta_{i} / \eta_{j}\right)^{\alpha} \eta$ are integrable for some $v \in \mathbb{R}^{n+1}$ and with $(\xi, \zeta)$ being infinitely divisible. Then $\mathrm{e}^{\xi}$ is quasi-swapinvariant of order $\alpha$ (i.e. satisfies (6.1) with $\vartheta=\langle v,(\xi, \zeta)\rangle)$ if and only if the characteristic triplet $(A, v, \gamma)$ of $(\xi, \zeta)$ satisfies the following conditions.

(a) If $n \geq 3$, the matrix A satisfies

$$
a_{l i}-a_{l j}=\frac{1}{2}\left(a_{i i}-a_{j j}\right)
$$

for all $l \neq i, j, l \leq n$.

(b) The image of $\hat{v} P^{-1}$ under $P$ of measure

$$
\mathrm{d} \hat{v}(x)=\mathrm{e}^{\left\langle v+(1+\alpha) e_{i} / 2+(1-\alpha) e_{j} / 2, x\right\rangle} \mathrm{d} v(x)
$$

is $\pi_{i j}$-invariant on $\mathbb{H} \backslash\{0\}$.

(c) $\gamma$ satisfies

$$
\begin{aligned}
\gamma_{i}-\gamma_{j}= & \frac{1}{2}\left(a_{j j}-a_{i i}\right)-\frac{\alpha}{2}\left(a_{i i}+a_{j j}-2 a_{i j}\right)+\sum_{k=1}^{n+1}\left(a_{j k}-a_{i k}\right) v_{k} \\
& +\int_{\mathbb{R}^{n+1}}\left(x_{j}-x_{i}\right)\left(\mathrm{e}^{\left\langle v+(1+\alpha) e_{i} / 2+(1-\alpha) e_{j} / 2, x\right\rangle} 1_{\{\|P x\| \leq 1\}}-1_{\{\|x\| \leq 1\}}\right) \mathrm{d} v(x) .
\end{aligned}
$$


For some applications, notably for semi-static hedging of barrier options with unequal carrying costs, the symmetry should be imposed on price changes adjusted with carrying costs. Unlike equity markets, where the assumption of equal carrying costs is often not totally unrealistic (e.g. in dividend-free cases), this assumption is quite restrictive in currency markets, since the risk-free interest rates in different countries usually differ. The carrying costs on various assets amount to componentwise multiplication of $\eta$ by a vector $\mathrm{e}^{\lambda}=\left(\mathrm{e}^{\lambda_{1}}, \ldots, \mathrm{e}^{\lambda_{n}}\right)$, where $\lambda_{i}=r-r_{i}, i=1, \ldots, n$. In currency trading $r_{i}$ denotes the risk-free interest rate in the $i$ th foreign market, while in the share case it becomes the dividend yield of the $i$ th share. If useful, $\lambda$ can also have other interpretations than being the pure carrying costs and $\eta$ need not be a one-period martingale itself.

Multiplying $\eta$ with a vector representing unequal carrying costs affects the (weighted) $i j$-swap-invariance property (4.1). However, in some cases it is possible to find $\alpha$ such that (6.1) holds, i.e. $\eta=\mathrm{e}^{\xi+\lambda}$ is quasi-swap-invariant. In this case $\xi+\lambda$ instead of $\xi$ satisfies (6.4). In the infinitely divisible case the only new condition on the Lévy triplet of $(\xi+\lambda, \zeta)$ concerns the 'drifts':

$$
\begin{aligned}
\gamma_{i}-\gamma_{j}= & \frac{1}{2}\left(a_{j j}-a_{i i}\right)-\frac{\alpha}{2}\left(a_{i i}+a_{j j}-2 a_{i j}\right)+\sum_{k=1}^{n+1}\left(a_{j k}-a_{i k}\right) v_{k} \\
& +\int_{\mathbb{R}^{n+1}}\left(x_{j}-x_{i}\right)\left(\mathrm{e}^{\left\langle v+(1+\alpha) e_{i} / 2+(1-\alpha) e_{j} / 2, x\right\rangle} 1_{\{\|P x\| \leq 1\}}-1_{\{\|x\| \leq 1\}}\right) \mathrm{d} v(x) \\
& +\lambda_{j}-\lambda_{i}
\end{aligned}
$$

Note that this condition depends only on the carrying costs of the $i$ th and $j$ th assets. If $\vartheta$ vanishes then the condition on the drift simplifies to

$$
\begin{aligned}
\gamma_{i}-\gamma_{j}= & \frac{1}{2}\left(a_{j j}-a_{i i}\right)-\frac{\alpha}{2}\left(a_{i i}+a_{j j}-2 a_{i j}\right) \\
& +\int_{\mathbb{R}^{n}}\left(x_{j}-x_{i}\right)\left(\mathrm{e}^{(1+\alpha) x_{i} / 2+(1-\alpha) x_{j} / 2} 1_{\left\{\left\|P^{\prime} x\right\| \leq 1\right\}}-1_{\{\|x\| \leq 1\}}\right) \mathrm{d} \nu(x)+\lambda_{j}-\lambda_{i} .
\end{aligned}
$$

Remark 6.1. (Determining $\alpha$ from the Lévy triplet and the carrying costs.) Consider $\eta=\mathrm{e}^{\xi+\lambda}$ that satisfies (6.1) with given $\lambda$ and $\vartheta=\zeta$ such that $(\xi, \zeta)$ is infinitely divisible. Note that neither (weighted) $i j$-swap-invariance nor the more general quasi-swap-invariance condition (6.1) implies that $\mathrm{Ee}^{\xi_{j}}=1$. Thus, for many applications, we additionally assume that $\mathrm{Ee}^{\xi_{l}}=$ $\varphi_{(\xi, \zeta)}\left(-\mathrm{i} e_{l}\right)=1$ for all $l$ and also that $\mathrm{E}^{\zeta}=1$. In particular, this implies that

$$
\gamma_{l}=-\frac{1}{2} a_{l l}-\int_{\mathbb{R}^{n+1}}\left(\mathrm{e}^{x_{l}}-1-x_{l} 1_{\{\|x\| \leq 1\}}\right) \mathrm{d} v(x), \quad l=i, j .
$$

Substitute (6.7) into (6.6) in order to see that $\alpha$ satisfies

$$
\begin{aligned}
\alpha\left(a_{i i}+\right. & \left.a_{j j}-2 a_{i j}\right) \\
= & 2\left(\lambda_{j}-\lambda_{i}\right)+2\left(a_{j(n+1)}-a_{i(n+1)}\right) \\
& +2 \int_{\mathbb{R}^{n+1}}\left(\mathrm{e}^{x_{i}}-\mathrm{e}^{x_{j}}+\left(x_{j}-x_{i}\right) \mathrm{e}^{\left\langle e_{n+1}+(1+\alpha) e_{i} / 2+(1-\alpha) e_{j} / 2, x\right\rangle} 1_{\{\|P x\| \leq 1\}}\right) \mathrm{d} v(x) .
\end{aligned}
$$

In the nonweighted case this condition can be written as

$$
\begin{aligned}
\alpha\left(a_{i i}+a_{j j}-2 a_{i j}\right)= & 2\left(\lambda_{j}-\lambda_{i}\right) \\
& +2 \int_{\mathbb{R}^{n}}\left(\mathrm{e}^{x_{i}}-\mathrm{e}^{x_{j}}+\left(x_{j}-x_{i}\right) \mathrm{e}^{(1+\alpha) x_{i} / 2+(1-\alpha) x_{j} / 2} 1_{\left\{\left\|P^{\prime} x\right\| \leq 1\right\}}\right) \mathrm{d} v(x) .
\end{aligned}
$$


In the Lévy processes setting the values of $\alpha$ calculated from the distributions at any time moment $t \geq 0$ coincide.

Example 6.1. (Black-Scholes setting.) In the absence of jumps it is easy to explicitly derive $\alpha$ from (6.8). Namely, if $v$ vanishes and $A$ satisfies (6.8), with $a_{i i}+a_{j j} \neq 2 a_{i j}$, then

$$
\alpha=2 \frac{\sum_{k=1}^{n+1}\left(a_{j k}-a_{i k}\right) v_{k}+\lambda_{j}-\lambda_{i}}{a_{i i}+a_{j j}-2 a_{i j}},
$$

which in the nonweighted case simplifies to

$$
\alpha=\frac{2\left(\lambda_{j}-\lambda_{i}\right)}{a_{i i}+a_{j j}-2 a_{i j}} .
$$

In the bivariate Black-Scholes case this result has been derived in [37] by directly using a symmetry result in univariate Black-Scholes markets. In Section 7 we show that this result can be used for semi-statically hedging certain generalised swap options in certain (in the bivariate case all) Black-Scholes economies.

Example 6.1 demonstrates that turning to the more general quasi-swap-invariance concept also in the equal carrying cost case $\left(\lambda_{i}=\lambda_{j}\right)$ yields considerably more flexibility for modelling the asset prices. In particular, for, e.g. $v=e_{3}$, each three-asset Black-Scholes model is quasiswap-invariant with $\alpha$ determined from (6.9).

\section{Hedging multi-asset barrier options}

In this section we show how the analysed symmetry properties can be used in order to create semi-static hedging strategies for several multi-asset options. First we derive in Section 7.1 a general hedging strategy for rather general options in (weighted) quasi-swap-invariant models, extending results obtained in [37], before applying them to well-known options in Section 7.2. We will also discuss examples where the more restrictive $i j$-exchangeability property is needed.

It should be noted that the suggested hedging strategies are only practicable if the instruments involved in the hedge are liquid or can be replicated by liquid instruments. Decompositions of not sufficiently liquid instruments in over-the-counter traded claims is an active area of current research and lies beyond the scope of this paper. In this relation, Carr and Laurence [13] wrote that 'all major banks stand ready to provide over-the-counter quotes on customised baskets' and mentioned a decomposition possibility of multivariate European payoff functions in basket options, thereby generalising the results of Lipton [28, Section 8.8] in the bivariate case; see also [8] and [25]. An easy decomposition formula for a large family of bivariate European payoff functions in other over-the-counter traded instruments is given in [38], while the results of [4] (in an appropriately adjusted interpretation) yield further decompositions in bivariate binary and certain bivariate correlation options. There is also a fast-growing literature about sub- and super-replication of basket options; see, e.g. [27], [33], and the literature cited therein. Furthermore, it is sometimes also possible to increase the liquidity of the involved instruments by implementing the hedges in a foreign derivative market or by using decomposition methods, similarly to [18] and [37]. Note that in special cases with equal (but not necessarily vanishing) carrying costs our hedging instruments are already of the form of exchange or basket options; see, e.g. the swap-invariant version of Example 7.2 or the nonquanto version of Example 7.3, respectively. 


\subsection{A general hedging strategy}

Consider a multivariate finite horizon model with the asset prices given by

$$
\left(S_{t}, Z_{t}\right)=\left(S_{0} \circ \mathrm{e}^{\tilde{\xi}_{t}}, Z_{0} \mathrm{e}^{\tilde{\zeta}_{t}}\right), \quad t \in[0, T],
$$

where $\left(\tilde{\xi}_{t}, \tilde{\zeta}_{t}\right)=\left(\lambda t+\xi_{t}, \mu t+\zeta_{t}\right)$ is a Lévy process such that all components of $\left(\mathrm{e}^{\xi_{t}}, \mathrm{e}^{\zeta_{t}}\right)$ are martingales defined on a filtered probability space $\left(\Omega, \mathfrak{F},\left(\mathfrak{F}_{t}\right)_{t \in[0, T]}, \mathrm{Q}\right)$ with the usual conditions imposed on the filtration. Note that the vector $(\lambda, \mu) \in \mathbb{R}^{n+1}$ represents deterministic carrying costs.

Fix $i<j, i, j \in\{1, \ldots, n\}$, and assume that, for every $t \in[0, T],\left(\mathrm{e}^{\tilde{\xi}_{t}}, \mathrm{e}^{\tilde{\zeta}_{t}}\right)$ satisfies the quasi-swap-invariance property (6.1) with $\eta=\mathrm{e}^{\tilde{\xi}_{t}}$ and $\vartheta=\tilde{\zeta}_{t}$, in particular, this involves the integrability of $Z_{t} S_{t}$ and $Z_{t} S_{t}\left(S_{t i} / S_{t j}\right)^{\alpha}$ for all $t \in[0, T]$.

Note that in real market applications often neither the 'symmetry assumption' nor the assumption that the asset prices follow multivariate componentwise exponentials of Lévy processes will typically be completely fulfilled. However, in the univariate case several comparative studies (see, e.g. [15], [19], and [32]) have confirmed a relatively good performance of symmetry-based semi-static hedges, even if the assumptions behind the semi-static hedges are not satisfied exactly. (We thank an anonymous referee for this hint.)

Consider a payoff function $g \in \mathcal{H}_{1}$ weighted by the terminal price $Z_{T}$ of the $(n+1)$ th asset, satisfying $\mathrm{E} Z_{T} g\left(S_{T}\right)<\infty$, with knock-in features given by the claims

$$
X=Z_{T} g\left(S_{T}\right) 1_{\{\tau \leq T\}},
$$

where

$$
\tau=\inf \left\{t: c S_{t i} \lesseqgtr S_{t j}\right\} .
$$

Note that we simultaneously handle the two knock-in cases corresponding to the crossing of the barrier from below or from above by the ratio process $S_{t j} / S_{t i}$, choosing the appropriate inequality in the indicator event. We assume that, for the crossing from below and above cases, the spot ratio $S_{0 j} / S_{0 i}$ lies below and, respectively, above the barrier.

Assume that the ratio process cannot jump over the barrier $c$. Then we can semi-statically replicate $X$ by the following (path-independent) European claim:

$$
G\left(S_{T}, Z_{T}\right)=Z_{T} g\left(S_{T}\right) 1_{B}+Z_{T} g\left(\hat{\kappa}\left(c, S_{T}\right)\right)\left(c \frac{S_{T i}}{S_{T j}}\right)^{\alpha} 1_{B_{0}} .
$$

Here $B=\left\{c S_{T i} \lesseqgtr S_{T j}\right\}, B_{0}=\left\{c S_{T i}>S_{T j}\right\}$, and

$$
\hat{\kappa}\left(c, S_{T}\right)=\left(S_{T 1}, \ldots, S_{T(i-1)}, \frac{S_{T j}}{c}, S_{T(i+1)}, \ldots, S_{T(j-1)}, c S_{T i}, S_{T(j+1)}, \ldots, S_{T n}\right) .
$$

In order to justify this hedge, note that on the event $\{\tau>T\}$ the claim in (7.1) expires worthless as desired. If the barrier knocks in then at time $\tau$ we can exchange (7.1) for a claim on $Z_{T} g\left(S_{T}\right)$ at zero costs. To confirm this, write

$$
Z_{T} g\left(S_{T}\right)=Z_{T} g\left(S_{T}\right) 1_{B}+Z_{T} g\left(S_{T}\right) 1_{B^{\mathrm{c}}},
$$

so we need to show that the conditional expectations of the second term on the right-hand side given the stopping $\sigma$-algebra $\mathfrak{F}_{\tau}$ coincides with the conditional expectation of the second term in (7.1) on the event $\{\tau \leq T\}$. 
Since $\left(\xi_{t}, \zeta_{t}\right)$ is a Lévy process, $\left(\left(\xi_{\tau}, \zeta_{\tau}\right),\left(\xi_{T}, \zeta_{T}\right)\right)$ and

$$
\left(\left(\xi_{\tau}, \zeta_{\tau}\right),\left(\xi_{\tau}+\xi_{T-\tau}^{\prime}, \zeta_{\tau}+\zeta_{(T-\tau)}^{\prime}\right)\right)
$$

share the same distribution on the event $\{\tau \leq T\}$, where $\left(\xi_{t}^{\prime}, \zeta_{t}^{\prime}\right), t \in[0, T]$, is an independent copy of the process $\left(\xi_{t}, \zeta_{t}\right), t \in[0, T]$. Hence, $\left(\left(S_{\tau}, Z_{\tau}\right),\left(S_{T}, Z_{T}\right)\right)$ and $\left(\left(S_{\tau}, Z_{\tau}\right)\right.$, $\left.\left(S_{\tau} \circ \eta_{\sigma}^{\prime}, Z_{\tau} Z_{\sigma}^{\prime}\right)\right)$ also coincide in distribution, where $\left(\eta_{t}^{\prime}, Z_{t}^{\prime}\right)$ is an independent copy of the process $\left(\eta_{t}, Z_{t}\right)$ and $\sigma=T-\tau$. The quasi-swap-invariance property (6.3) together with Remark 5.4 yields

$$
\begin{aligned}
\mathrm{E}\left(Z_{T} g\left(S_{T}\right) 1_{B^{c}} \mid \mathfrak{F}_{\tau}\right) & =\mathrm{E}\left(Z_{\tau} Z_{\sigma}^{\prime} g\left(S_{\tau} \circ \eta_{\sigma}^{\prime}\right) 1_{\left\{c S_{\tau i} \eta_{\sigma i<}^{\prime} S_{\tau j} \eta_{\sigma j}^{\prime}\right\}} \mid \mathfrak{F}_{\tau}\right) \\
& =\mathrm{E}\left(Z_{\tau} Z_{\sigma}^{\prime} g\left(S_{\tau} \circ \pi_{i j} \eta_{\sigma}^{\prime}\right) 1_{\left\{c S_{\tau i} \eta_{\sigma j<}^{\prime}>S_{\tau j} \eta_{\sigma i}^{\prime}\right\}}\left(\frac{\eta_{\sigma i}^{\prime}}{\eta_{\sigma j}^{\prime}}\right)^{\alpha} \mid \mathfrak{F}_{\tau}\right) \\
& =\mathrm{E}\left(Z_{\tau} Z_{\sigma}^{\prime} g\left(\hat{\kappa}\left(c, S_{\tau} \circ \eta_{\sigma}^{\prime}\right)\right) 1_{\left\{S_{\tau j} \eta_{\sigma j<}^{\prime}>c S_{\tau i} \eta_{\sigma i}^{\prime}\right\}}\left(\frac{c S_{\tau i} \eta_{\sigma i}^{\prime}}{S_{\tau_{j}} \eta_{\sigma j}^{\prime}}\right)^{\alpha} \mid \mathfrak{F}_{\tau}\right) \\
& =\mathrm{E}\left(Z_{T} g\left(\hat{\kappa}\left(c, S_{T}\right)\right)\left(c \frac{S_{T i}}{S_{T j}}\right)^{\alpha} 1_{B_{0}} \mid \mathfrak{F}_{\tau}\right)
\end{aligned}
$$

on the event $\{\tau \leq T\}$. Note that we have used the fact that $S_{\tau j}=c S_{\tau i}$. Hence, on the event $\{\tau \leq T\}$

$$
\mathrm{E}\left(Z_{T} g\left(S_{T}\right) \mid \mathfrak{F}_{\tau}\right)=\mathrm{E}\left(G\left(S_{T}, Z_{T}\right) \mid \mathfrak{F}_{\tau}\right)
$$

The above arguments also can be used to valuate the described barrier options for models with continuous sample paths in the $i$ th and $j$ th components.

Note that if the ratio process can jump over the barrier, the hedge in (7.1) is no longer exact.

\subsection{Illustrative examples}

We will assume in all examples without loss of generality that $i=1$ and $j=2$, and so accordingly speak about 12-swap-invariance or 12-exchangeability.

Example 7.1. (Barrier quanto-swap options.) Consider a vector of asset prices

$$
S_{t}=\left(S_{t 1}, S_{t 2}, S_{t 3}\right)=\left(S_{01} \mathrm{e}^{\lambda_{1} t} \mathrm{e}^{\xi_{t 1}}, S_{02} \mathrm{e}^{\lambda_{2} t} \mathrm{e}^{\xi_{t 2}}, S_{03} \mathrm{e}^{\lambda_{3} t} \mathrm{e}^{\xi_{t 3}}\right)=S_{0} \circ \mathrm{e}^{\xi_{t}+\lambda t}
$$

with $\lambda=\left(\lambda_{1}, \lambda_{2}, \lambda_{3}\right)$ representing the carrying costs and

$$
\eta_{t}=\left(\eta_{t 1}, \eta_{t 2}, \eta_{t 3}\right)=\left(\mathrm{e}^{\lambda_{1} t+\xi_{t 1}}, \mathrm{e}^{\lambda_{2} t+\xi_{t 2}}, \mathrm{e}^{\lambda_{3} t+\xi_{t 3}}\right)=\mathrm{e}^{\xi_{t}+\lambda t}
$$

being 12-quasi-swap-invariant (with the weight given by the third asset) for all $t \in[0, T]$, where other conditions remain the same as in Section 7.1. Consider barrier claims defined by

$$
\begin{aligned}
X_{\mathrm{qsw}} & =S_{T 3}\left(a S_{T 1}-b S_{T 2}\right)_{+} 1_{\left\{\text {there exists } t \in[0, T], c S_{t 1} \leq S_{t 2}\right\}}, \\
Y_{\mathrm{qsw}} & =S_{T 3}\left(a S_{T 1}-b S_{T 2}\right)_{+} 1_{\left\{c S_{t 1}>S_{t 2} \text { for all } t \in[0, T]\right\}},
\end{aligned}
$$

where $c S_{01}>S_{02}$, the positive parameters satisfy $0<a \leq b c$, and

$$
\tau=\inf \left\{t: c S_{t 1} \leq S_{t 2}\right\}
$$


By (7.1), the hedge portfolio for $X_{\mathrm{qsw}}$ is given by

$$
\begin{aligned}
G\left(S_{T 1}, S_{T 2}, S_{T 3}\right)= & S_{T 3}\left(a S_{T 1}-b S_{T 2}\right)_{+} 1_{\left\{c S_{T 1} \leq S_{T 2}\right\}} \\
& +S_{T 3}\left(\frac{a}{c} S_{T 2}-b c S_{T 1}\right)_{+}\left(c \frac{S_{T 1}}{S_{T 2}}\right)^{\alpha} 1_{\left\{c S_{T 1}<S_{T 2}\right\}} .
\end{aligned}
$$

Since $0<a \leq b c$, if $c S_{T 1} \leq S_{T 2}$ then $\left(a S_{T 1}-b S_{T 2}\right)_{+}$is out of the money, so the first term vanishes. Furthermore, $0<a \leq b c$ implies that if $\left(a S_{T 2} / c-b c S_{T 1}\right)_{+}$is (strictly) positive then $c S_{T 1}<S_{T 2}$, so we can omit the indicator function in the second term. Thus, $X_{\mathrm{qsw}}$ can be hedged (exactly if the ratio process cannot jump over the barrier) with a long position in the European derivative with payoff

$$
S_{T 3}\left(\frac{a}{c} S_{T 2}-b c S_{T 1}\right)_{+}\left(c \frac{S_{T 1}}{S_{T 2}}\right)^{\alpha} .
$$

By the knock-in-knock-out parity, $Y_{\mathrm{qsw}}$ can be hedged by a short position in this derivative and a long position in the European derivative with payoff $S_{T 3}\left(a S_{T 1}-b S_{T 2}\right)_{+}$. In the weighted 12-swap-invariant case $\alpha=0$ and the hedging instruments reduce to weighted quanto-swap options.

Example 7.2. (Barrier swap options.) By specialising the claims $X_{\mathrm{qsw}}$ and $Y_{\mathrm{qsw}}$ to the cases where $S_{T 3}=1$ (with the other assumptions unchanged), we get weighted barrier swap (also known as Margrabe) options with knocking conditions, defined by the claims

$$
\begin{aligned}
X_{\mathrm{SW}} & =\left(a S_{T 1}-b S_{T 2}\right)_{+} 1_{\left\{\text {there exists } t \in[0, T], c S_{t 1} \leq S_{t 2}\right\}}, \\
Y_{\mathrm{sW}} & =\left(a S_{T 1}-b S_{T 2}\right)_{+} 1_{\left\{c S_{t 1}>S_{t 2} \text { for all } t \in[0, T]\right\}},
\end{aligned}
$$

where the assumptions on the parameters $a, b$, and $c$ remain unchanged. Assuming that $\left(\eta_{t 1}, \eta_{t 2}\right)$ is quasi-swap-invariant, the hedging portfolio for $X_{\mathrm{sw}}$ consists of a long position in the European derivative with payoff $\left(a S_{T 2} / 2-b c S_{T 1}\right)_{+}\left(c S_{T 1} / S_{T 2}\right)^{\alpha}$, while the hedge of $Y_{\mathrm{sw}}$ is given by a short position in this derivative and a long position in the European derivative with payoff $\left(a S_{T 1}-b S_{T 2}\right)_{+}$. In the swap-invariant case all hedging instruments reduce to weighted swap options and weighted Margrabe options, respectively.

Example 7.3. (Hedges based on the exchangeability property.) We end this section by discussing an example where we need more symmetry in the model than (weighted) swap invariance in order to hedge some basket payoffs with barrier features on a ratio process. Assume that the vector of asset prices from Example 7.1, $S_{t}=\left(S_{t 1}, S_{t 2}, S_{t 3}\right)$, is such that (e $\left.\mathrm{e}^{\xi_{t 1}}, \mathrm{e}^{\xi_{t 2}}, \mathrm{e}^{\xi_{t 3}}\right)$ is 12-exchangeable for all $t \in[0, T]$, while the remaining assumptions are not changed. Let the carrying costs $\lambda_{1}=\lambda_{2}$ be the same for the first and the second assets, e.g. both being the risk-free interest rate. Assume that $c S_{01}>S_{02}$, and define the stopping time $\tau$ by (7.2). Consider the claim

$$
Y_{\mathrm{qsp}}=S_{T 3}\left(a S_{T 1}-b S_{T 2}-k\right)_{+} 1_{\left\{c S_{t 1}>S_{t 2} \text { for all } t \in[0, T]\right\}},
$$

with positive weights $a, b, c, a \leq b c$ and nonnegative strike $k$. This option is knocked out if the ratio $S_{t 2} / S_{t 1}$ achieves or exceeds $c$.

We again assume for the moment that jumps cannot cross the barrier, e.g. as in the case of the Black-Scholes setting. By similar arguments as in Example 7.1, we can hedge the claim 
$Y_{\mathrm{qsp}}$ by taking the following positions in the European weighted quanto-spread options:

$$
\begin{array}{ll}
\text { long } & S_{T 3}\left(a S_{T 1}-b S_{T 2}-k\right)_{+}, \\
\text {short } & S_{T 3}\left(\frac{a}{c} S_{T 2}-b c S_{T 1}-k\right)_{+} .
\end{array}
$$

This also yields the result that the knock-in claim

$$
X_{\mathrm{qsp}}=S_{T 3}\left(a S_{T 1}-b S_{T 2}-k\right)_{+} 1_{\left\{\text {there exists } t \in[0, T], c S_{t 1} \leq S_{t 2}\right\}},
$$

with the same parameters $a, b, c$, and $k$, can be hedged with a long position in the European option given by the payoff function

$$
S_{T 3}\left(\frac{a}{c} S_{T 2}-b c S_{T 1}-k\right)_{+} .
$$

In the case of jump processes the exchangeability implies that $\mathrm{e}^{\xi_{t 1}}$ has up or down jumps if and only if $\mathrm{e}^{\xi_{t 2}}$ has up or, respectively, down jumps, so that $c S_{\tau 1}$ is no longer almost surely equal $S_{\tau 2}$. This fact leads to a super-replication of knock-in options and a sub-replication of knock-out options.

\section{Acknowledgements}

The authors are grateful to the anonymous referees for carefully reading this paper and suggesting improvements.

\section{References}

[1] Aldous, D. J. (1985). Exchangeability and Related Topics (Lecture Notes Math 1117). Springer, Berlin.

[2] Andersen, L. B. G., Andreasen, J. And Eliezer, D. (2002). Static replication of barrier options: some general results. J. Comput. Finance 5, 1-25.

[3] Andreasen, J. (2001). Behind the mirror. Risk 14, 108-110.

[4] Baldeaux, J. and Rutkowski, M. (2010). Static replication of forward-start claims and realized variance swaps. Appl. Math. Finance 17, 99-131.

[5] Barndorff-Nielsen, O. E. (1977). Exponentially decreasing distributions for the logarithm of particle size. Proc. R. Soc. London A 353, 401-419.

[6] Bates, D. S. (1991). The crash of '87: was it expected? The evidence from options markets. J. Finance 46, 1009-1044.

[7] Bates, D. S. (1997). The skewness premium: option pricing under asymmetric processes. Adv. Futures Options Res. 9, 51-82.

[8] BaXter, M. (1998). Hedging in financial markets. ASTIN Bull. 28, 5-16.

[9] BowIE, J. AND CARr, P. (1994). Static simplicity. Risk 7, 45-49.

[10] Breeden, D. T. and Litzenberger, R. H. (1978). Prices of state-contingent claims implicit in options prices. J. Business 51, 621-651.

[11] CARr, P. (1994). European put call symmetry. Tech. Rep., Cornell University.

[12] CARr, P. And Chou, A. (1997). Breaking barriers. Risk 10, 139-145.

[13] Carr, P. and Laurence, P. (2010). Multi-asset stochastic local variance contracts. Math. Finance 21, 21-52.

[14] Carr, P. And Lee, R. (2009). Put-call symmetry: extensions and applications. Math. Finance 19, 523-560.

[15] CARR, P. AND Wu, L. (2006). Hedging barriers. Tech. Rep., Bloomberg LP and Baruch College.

[16] Carr, P., Ellis, K. and Gupta, V. (1998). Static hedging of exotic options. J. Finance 53, 1165-1190.

[17] Eberlein, E., Papapantoleon, A. and Shiryaev, A. N. (2008). On the duality principle in option pricing: semimartingale setting. Finance Stoch. 12, 265-292.

[18] Eberlein, E., Papapantoleon, A. and Shiryaev, A. N. (2009). Esscher transform and the duality principle for multidimensional semimartingales. Ann. Appl. Prob. 19, 1944-1971. 
[19] Engelmann, B., Fengler, M. R., Nalholm, M. and Schwendner, P. (2006). Static versus dynamic hedges: an empirical comparison for barrier options. Rev. Derivataives Res. 9, 239-264.

[20] Fajardo, J. ANd Mordecki, E. (2006). Pricing derivatives on two-dimensional Lévy processes. Internat. J. Theoret. Appl. Finance 9, 185-197.

[21] Fajardo, J. and Mordecki, E. (2006). Skewness premium with Lévy processes. Working Paper, IBMEC.

[22] Fajardo, J. And Mordecki, E. (2006). Symmetry and duality in Lévy markets. Quant. Finance 6, $219-227$.

[23] Fajardo, J. and Mordecki, E. (2008). Duality and derivative pricing with time-changed Lévy processes. Brazilian Rev. Econometrics 28, 95-110.

[24] Grabbe, J. O. (1983). The pricing of call and put options on foreign exchange. J. Internat. Money Finance 2, 239-253.

[25] Henkin, G. M. and Shananin, A. A. (1990). Bernstein theorems and Radon transform. Application to the theory of production functions. In Mathematical Problems of Tomography, eds I. M. Gel'fand and S. G. Gindikin, American Mathematical Society, Providence, RI, pp. 189-223.

[26] Kallenberg, O. (2005). Probabilistic Symmetries and Invariance Principles. Springer, New York.

[27] Laurence, P. And WAng, T.-H. (2009). Sharp distribution free lower bounds for spread options and the corresponding optimal subreplicating portfolios. Insurance Math. Econom. 44, 35-47.

[28] Lipton, A. (2001). Mathematical Methods for Foreign Exchange. World Scientific, River Edge, NJ.

[29] Masuda, H. (2004). On multidimensional Ornstein-Uhlenbeck processes driven by a general Lévy process. Bernoulli 10, 97-120.

[30] Molchanov, I. ANd Schmutz, M. (2010). Multivariate extension of put-call symmetry. SIAM J. Financial Math. 1, 396-426.

[31] Mosler, K. (2002). Multivariate Dispersion, Central Regions and Depth. The Lift Zonoid Approach (Lecture Notes Statist. 165). Springer, Berlin.

[32] Nalholm, M. And Poulsen, R. (2006). Static hedging and model risk for barrier options. J. Futures Markets 26, 449-463.

[33] Peña, J., Saynac, X., Vera, J. C. And Zuluaga, L. (2010). Computing general static-arbitrage bounds for European basket options via Dantzig-Wolfe decomposition. Algorithmic Operat. Res. 5, 65-74.

[34] Ross, S. A. (1976). Options and efficiency. Quart. J. Econom. 90, 75-89.

[35] Sato, K.-I. (1999). Lévy Processes and Infinitely Divisible Distributions. Cambridge University Press.

[36] Sato, K.-I. (2000). Density transformation in Lévy processes. MaPhySto Lecture Notes, Vol. 7, Aarhus University.

[37] Schmutz, M. (2011). Semi-static hedging for certain Margrabe type options with barriers. Quant. Finance 11, 979-986.

[38] Schmutz, M. And Zürcher, T. (2010). Static replications with traffic light options. Tech. Rep., University of Bern. Available at http://arxiv.org/abs/1011.4795v1.

[39] Tehranchi, M. R. (2009). Symmetric martingales and symmetric smiles. Stoch. Process. Appl. 119, 3785-3797.

[40] Von Hammerstein, E. A. (2004). Lévy-Khintchine representation of multivariate generalized hyperbolic distributions and some of their limiting cases. Tech. Rep., University of Freiburg. 